\title{
ZOOLOGÍA
}

\section{MAMÍFEROS (MAMMALIA) DEL DEPARTAMENTO DE PUTUMAYO, COLOMBIA}

\author{
Héctor E. Ramírez-Chaves*, Elkin A. Noguera-Urbano**, Miguel E. Rodríguez-Posada***
}

\begin{abstract}
RESUMEN
Ramírez-Chaves H. E., E. A. Noguera-Urbano, M. E. Rodríguez-Posada: Mamíferos (Mammalia) del departamento de Putumayo, Colombia. Rev. Acad. Colomb. Cienc. 37 (143): 263-286, 2013. ISSN 0370-3908.

Presentamos información sobre la riqueza, distribución e investigación mastozoológica en el departamento de Putumayo. Un total de 154 especies distribuidas en 13 órdenes y 37 familias son registradas para el departamento. Chiroptera, Rodentia, Carnivora y Primates fueron los órdenes más diversos con 66, 24, 19 y 15 especies respectivamente. Los distritos biogeográficos con mayor riqueza de especies fueron el Distrito Alto Putumayo con 136, mientras que la eco-región con mayor riqueza fue la Selva Húmeda del Napo con 131 especies. El municipio con mayor número de especies registradas fue Puerto Leguízamo con 100. Resaltamos la ausencia de estudios regionales y el escaso conocimiento generado en este sector de Colombia.
\end{abstract}

Palabras clave: colectores, distribución, historia, mamíferos, riqueza.

\begin{abstract}
We present information regarding to the richness, distribution and mammalogical research in the Department of Putumayo. A total of 154 species of 13 orders and 37 families were registered in the Department. Chiroptera, Rodentia, Carnivora and Primates were the most diverse orders with 66, 24, 19 and 15 species. The Alto Putumayo biogeographic District presents the highest richness with 136 species, while the Napo Humid Forest was the richest Ecoregion with 131 species. Puerto Leguízamo was the Municipality with the highest number of species with 100 . We highlighted the absence of regional studies and the poor knowledge associated to mammals, generated in this sector of Colombia.
\end{abstract}

Key words: collectors, distribution, history, mammals, richness.

* Weisbecker Lab, University of Queensland, School of Biological Sciences, Goddard Building 8, St. Lucia 4072, Australia. Correo electrónico: hera.chaves@gmail.com

** Museo de Zoología “Alfonso L. Herrera”, Departamento de Biología Evolutiva, Facultad de Ciencias, Universidad Nacional Autónoma de México. Apartado Postal 70-399, 04510 México, D. F., México. Correo electrónico: elkalexno@gmail.com.

*** Laboratorio de Mamíferos, Facultad de Ciencias, Instituto de Ciencias Naturales, Universidad Nacional de Colombia, Carrera 45 \#26-85, Sede Bogotá D.C., Colombia. Correo electrónico: migrodriguezp@yahoo.com 


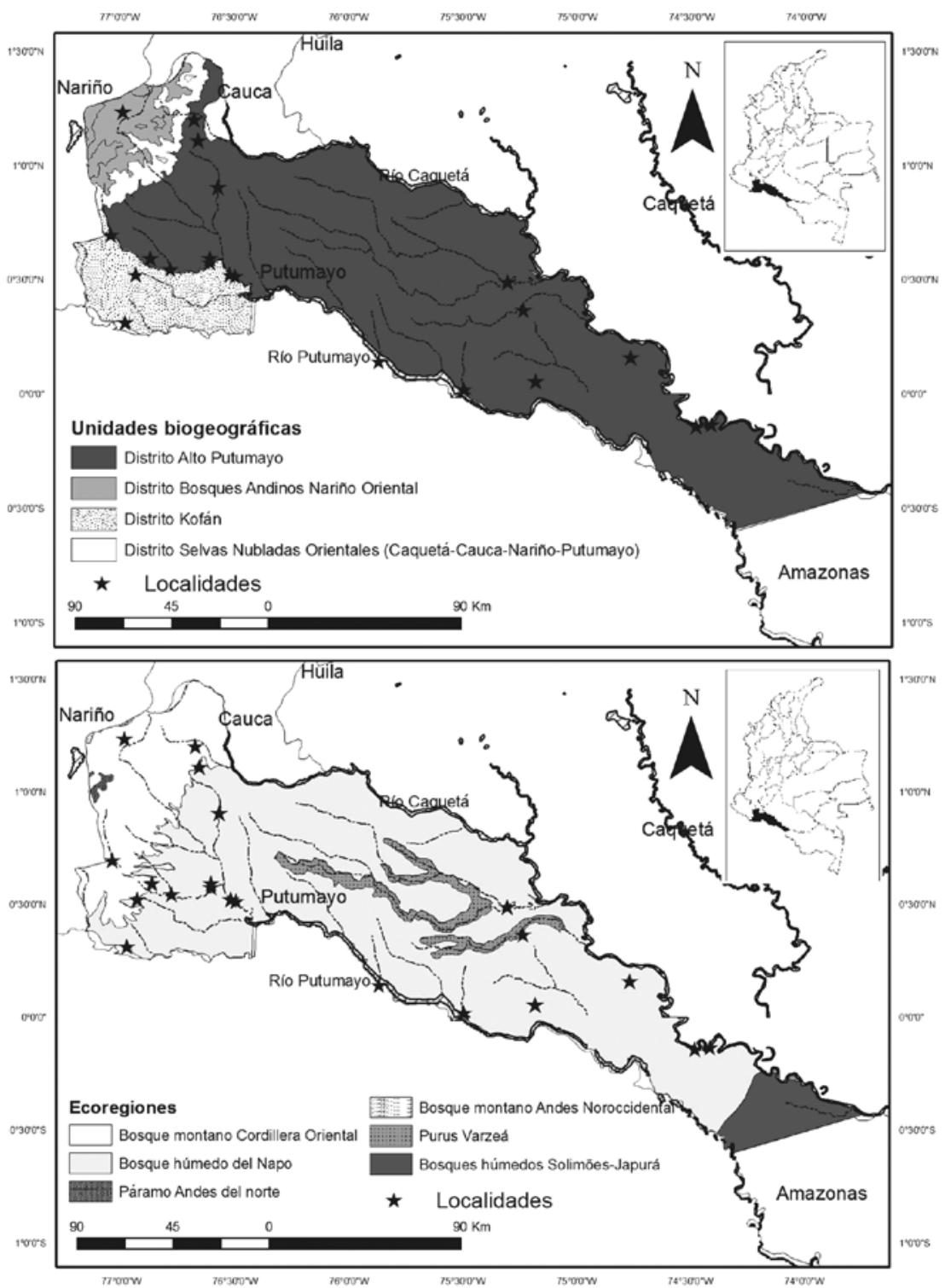

Figura 1. Localización del departamento de Putumayo. Los puntos negros indican las localidades de los registros. El mapa superior indica los distritos biogeográficos según Hernández-Camacho et al., (1992a), el Distrito Páramos (Doña Juana) no se aprecia por la escala del mapa. El mapa inferior indica los tipos de eco-regiones según Olson et al., (2001).

\section{Introducción}

El departamento de Putumayo presenta una extensión de $24885 \mathrm{Km}^{2}$ y se localiza en el suroriente de Colombia ( $01^{\circ} 26^{\prime}$ y $00^{\circ} 27^{\prime} \mathrm{N}$ y $\operatorname{los} 73^{\circ} 50^{\prime}$ y $\left.77^{\circ} 04^{\prime} \mathrm{W}\right)$. Limita al norte con los departamentos del Cauca y Caquetá, al oriente con el departamento de Caquetá, al sur con el departamento de Amazo- nas y las Repúblicas del Ecuador (Provincia de Sucumbíos) y Perú (departamento de Loreto) y al occidente con el departamento de Nariño. La mayor parte del territorio corresponde a tierras bajas con altitudes por debajo de los $300 \mathrm{~m}$, sin embargo el extremo occidental del departamento se ubica sobre el flanco oriental de la cordillera de los Andes, donde alcanza altitudes superiores a los $3800 \mathrm{~m}$ (IGAC, 1996). 
El departamento de Putumayo presenta diferentes ecosistemas que comprenden selvas bajas y de piedemonte amazónico hasta páramos (Mueses-Cisneros, 2005). Se han propuesto dos divisiones biogeográficas del departamento. Hernández-Camacho et al., (1992a), propusieron cinco distritos: (1) Bosques Andinos Nariño oriental; (2) Bosques de niebla orientales; (3) Páramos Doña Juana; (4) Alto Putumayo y (5) Distrito Kofán (Figura 1a). Por otra parte, Olson et al., (2001) identificaron seis eco-regiones: (1) Bosque montano Andes noroccidental-Doña Juana; (2) Paramo Norandino-Valle de Sibundoy; (3) Bosque montano de la cordillera Oriental-Mocoa; (4) Selva húmeda del Napo; (5) Purus Várzea-áreas inundables; y el (6) Bosque húmedo Solimões-Japurá (Figura 1b). En su territorio existen tres áreas protegidas por el Sistema Nacional de Áreas Protegidas (SINAP): el Parque Nacional Natural (PNN) La Paya, localizado en el municipio de Puerto Leguízamo; el PNN Serranía de Los Churumbelos Auka-Wasi, en el municipio de Mocoa (Putumayo), pero que se extiende hacia los departamentos de Caquetá, Cauca y Huila, y el Santuario de Flora Plantas Medicinales (SFPM) Orito Ingi-Ande, en el municipio de Orito, que se extiende hacia los municipio de Pasto y Funes del departamento de Nariño (Polanco-Ochoa et al., 2000; PNN, 2011).

La fauna de mamíferos del departamento de Putumayo ha sido poco documentada; sólo existe un estudio publicado que corresponde a una evaluación ecológica rápida realizada en el PNN La Paya (Polanco-Ochoa et al., 2000); otra información sobre los mamíferos del departamento está dispersa en la literatura que comprende revisión de especímenes de diferentes localidades. Es importante destacar que dos especies presentan su localidad típica en el departamento Diclidurus ingens Hernández-Camacho, 1955 y Callicebus medemi Hershkovitz, 1963.

Los primeros registros de mamíferos de Putumayo son anecdóticos y datan de la época de la conquista, cuando Fray Juan de Santa Gertrudis (1956 [1775]) mencionó la presencia de algunos felinos, hormigueros, armadillos, primates e incluso delfines en su viaje por el río Putumayo. A partir de mediados del siglo XX algunos naturalistas e investigadores exploraron la región, recolectando especímenes de estudio que actualmente se encuentran depositados en colecciones científicas nacionales e internacionales, sin embargo, en nuestro conocimiento no existe una compilación que permita ubicar y cuantificar este material.

El objetivo del presente trabajo es consolidar la información disponible sobre la fauna de mamíferos del departamento de Putumayo. Para esto presentamos el estado de conocimiento de los mamíferos presentes en el departamento, una lista anotada actualizada de las especies que la componen y anali- zamos su distribución con base a las dos propuestas biogeográficas citadas. Adicionalmente, analizamos los registros disponibles a nivel político-administrativo y señalamos los vacíos de información.

\section{Materiales y métodos}

\section{Estado del conocimiento}

Revisamos publicaciones científicas y consultamos bases de datos de museos nacionales e internacionales a través del portal del sistema de información de mamíferos en red MaNIS (2012) (http://manisnet.org). De estas bases de datos recompilamos información de aproximadamente 1600 ejemplares de referencia, procedentes del departamento de Putumayo. A partir de esta información, mencionamos los colectores que más aportaron y los años en los que se hicieron los mayores esfuerzos de muestreo, para analizar cómo ha sido el avance de la investigación mastozoológica en esta región. Incluimos un análisis donde se relaciona el número de trabajos y el grupo taxonómico a nivel de orden, así como el número de ejemplares disponibles de los diferentes órdenes de mamíferos registrados, depositados las siguientes colecciones consultadas: Instituto Alexander von Humboldt (IAvH); Instituto de Ciencias Naturales, Universidad Nacional de Colombia (ICN); Colección zoológica de Referencia del Museo de Ciencias Naturales Federico Carlos Lehmann V. (IMCN); Museo de Historia Natural-Universidad del Cauca (MHNUC); Colección Zoológica Universidad de Nariño (PSO-CZ), Muséum d'Histoire Naturelle (MHNG). Adicionalmente, consultamos las bases de datos de las siguientes colecciones norteamericanas disponibles en MaNIS (2012): American Museum of Natural History (AMNH), Field Museum of Natural History (FMNH), University of Kansas Biodiversity Research Center (KU), Museum of Vertebrate Zoology (MVZ), Royal Ontario Museum (ROM), Texas Cooperative Wildlife Collection (TCWC), United States National Museum, Smithsonian Institution (USNM).

\section{Lista de especies}

La lista anotada fue elaborada a partir de la información recopilada. Igualmente incluimos información sobre capturas, observaciones, entrevistas y registros fotográficos recolectada por los autores.

Seguimos el tratamiento taxonómico propuesto por Wilson \& Reeder (2005), con las modificaciones realizadas por Velazco (2005), Velazco \& Gardner (2009) y Velazco et al., (2010) para el género Platyrrhinus, Weksler et al., (2006) para los roedores Oryzominos y Rossi et al., (2010) para el complejo Marmosa 'mexicana'. 
Tabla 1. Especies amenazadas de mamíferos que se reportan en el departamento de Putumayo.

\begin{tabular}{|c|l|l|l|c|c|}
\hline N$^{\circ}$ & Nombre común & Orden & Especie & $\begin{array}{c}\text { Rodríguez-Ma- } \\
\text { hecha et al. 2006 }\end{array}$ & IUCN 2012 \\
\hline 1 & Tigrillo & Carnivora & Leopardus tigrinus & VU & VU \\
\hline 2 & Nutría o lobito de río & Carnivora & Lontra longicaudis & VU & DD \\
\hline 3 & Tigre, Jaguar & Carnivora & Panthera onca & VU & NT \\
\hline 4 & Zorro paramuno & Carnivora & Lycalopex culpaeus & VU & LC \\
\hline 5 & Nutria & Carnivora & Pteronura brasiliensis & EN & EN \\
\hline 6 & Oso de anteojos & Carnivora & Tremarctos ornatus & VU & VU \\
\hline 7 & Delfín de río, bufeo & Cetacea & Inia geoffrensis & VU & DD \\
\hline 8 & Delfín & Cetacea & Sotalia fluviatilis & VU & DD \\
\hline 9 & Danta de páramo & Perissodactyla & Tapirus pinchaque & EN & EN \\
\hline 10 & Danta, sachavaca & Perissodactyla & Tapirus terrestris & CR & VU \\
\hline 11 & Mono araña & Primates & Ateles belzebuth & VU & EN \\
\hline 12 & Socay o zocay & Primates & Callicebus discolor & VU & LC \\
\hline 13 & Socay o zocay & Primates & Callicebus medemi & No evaluada & VU \\
\hline 14 & Chichico negro & Primates & Callimico goeldii & VU & VU \\
\hline 15 & Huapo negro & Primates & Pithecia monachus & VU & LC \\
\hline 16 & Mono chorongo & Primates & Lagothrix lagothricha & VU & VU \\
\hline 17 & Guagua loba & Rodentia & Dinomys branickii & VU & VU \\
\hline 18 & Manatí & Sirenia & Trichechus inunguis & EN & VU \\
\hline 19 & Hormiguero & Vermilingua & Myrmecophaga tridactyla & VU & VU \\
\hline
\end{tabular}

Los registros de los ejemplares con los municipios y localidades de procedencia se incluyen en la lista de especies. Para la categorización de las especies amenazadas seguimos a Rodríguez-Mahecha et al., (2006a) y la Unión Internacional para la conservación de la Naturaleza -IUCN (2012) (Tabla 1).

\section{Distribución}

Con el fin explorar de manera preliminar la distribución de los registros y especies de mamíferos en relación a la división política del departamento, a los distritos biogeográficos de Hernández-Camacho et al., (1992a) y las eco-regiones de Olson et al., (2001), estimamos el número de especies y registros por unidad; para ello hicimos coincidir las localidades con mapas de los tres tipos (administrativo, distritos biogeográficos y eco-regiones). Procesamos 321 registros y compilamos 55 localidades del departamento de las cuales 47 son precisas e informativas por su detalle en la ubicación, para 146 especies de mamíferos con distribución en el Putumayo. Excluimos las ocho localidades no informativas que provienen de 13 registros e igual número de especies (mencionadas en Cuervo et al., 1986; Tamsitt et al., 1986, Rodríguez-Mahecha et al., 1995, 2006; Alberico et al.,

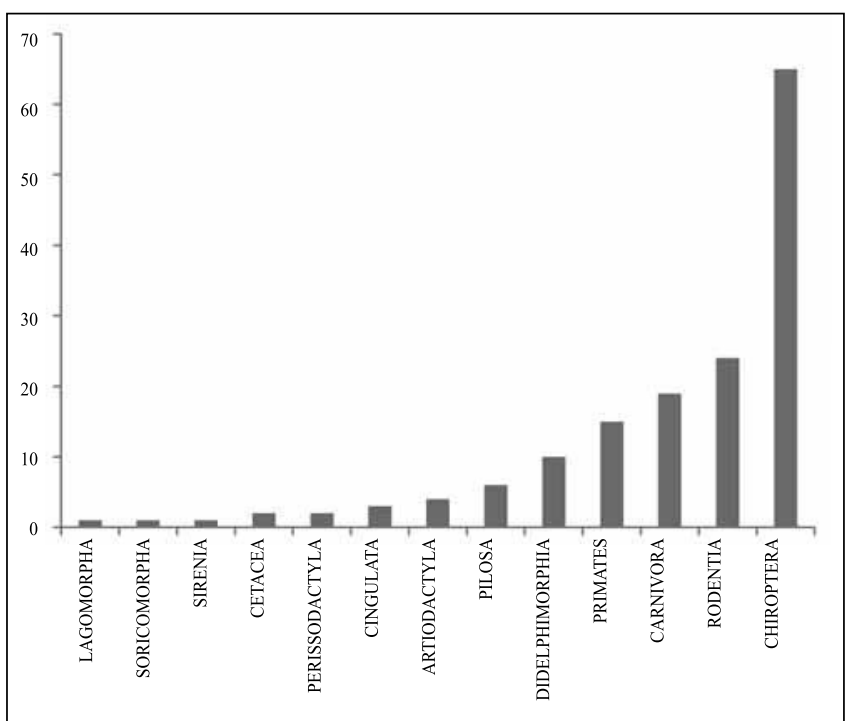

Figura 2. Número de menciones en literatura para los órdenes de mamíferos registrados hasta la fecha en el departamento de Putumayo.

2000, Mantilla-Meluk et al., 2009), porque consistían en solo la mención del departamento o el nombre de un río, la 
orilla o banco de un cuerpo hídrico, sin asociar información sobre el municipio o vereda; la ambigüedad de dichas localidades no permitió definirlas en ninguno de los dos tipos de clasificación biogeográfica utilizadas en este estudio.

\section{Resultados}

\section{Estado del conocimiento}

Los mayores esfuerzos para conocer la fauna de mamíferos de Putumayo empezaron en 1952, cuando Philip Hershkovitz recolectó más de 200 especímenes para el FMNH, en el río Mecaya. Posteriormente Arturo Pazos recolectó una de las series más grandes de pequeños mamíferos procedente de Putumayo (más de 140 ejemplares) para el ROM entre 1964 y 1972. En 1965, B. Mechler recolectó 88 especímenes, principalmente de murciélagos, en las localidades de Mocoa, Orito y Puerto Asís, mientras que Werren y Mengolli recolectaron en Puerto Asís ese mismo año, para el MHNG. Entre 1965 y 1966 Herve le Nestour recolectó ejemplares en el bajo río Guamués, cerca de Puerto Asís para el ICN en Bogotá (alrededor de 69 ejemplares) y para el MHNG (alrededor de once especímenes). Entre los años 1968-1969, Cornelius J. Marinkelle recolectó especímenes en la localidad de Leguízamo y estas colectas se encuentran depositadas en el ICN y el ROM. John A. W. Kirsch recolectó especíme- nes en 1969 en Puerto Asís, para la KU. Entre 1969-1971, Kjell von Sneidern recolectó ejemplares en San Antonio, El Carmen y el río Guamués, para el ICN, FMNH y el MNHG; las colecciones para el FMNH superan los 400 individuos. Entre 1970 y 1971, B. Malkin recolectó ejemplares en río San Miguel para el FMNH. En 1994, Rocío Polanco-Ochoa recolectó 85 ejemplares en el PNN La Paya, los cuales se encuentran depositados en el ICN.

La mayor cantidad de ejemplares de referencia de Putumayo se encuentran depositados en colecciones extranjeras entre las que se destaca el FMNH, con más de 460 ejemplares de los órdenes Chiroptera, Rodentia y Primates (Figura 3), seguido por el ROM con 434 ejemplares de los órdenes Chiroptera, Didelphimorphia, Rodentia, Lagomorpha y Pilosa (Figura 3). De las colecciones colombianas, el ICN posee aproximadamente 208 ejemplares, con mayor representatividad de los órdenes Chiroptera, Rodentia, Primates, Carnivora y Artiodactyla (Figura 3), mientras que el IAvH posee alrededor de 45 ejemplares principalmente de Chiroptera, Didelphimorphia y Rodentia (Figura 3).

Los resultados de las recolectas realizadas por PolancoOchoa fueron publicados en la única contribución sobre mamíferos de Putumayo (Polanco-Ochoa et al., 2000), en la cual se registran 77 especies, 32 de pequeños mamíferos pequeños, 45 de medianos y grandes, y el uso dado a 26 de

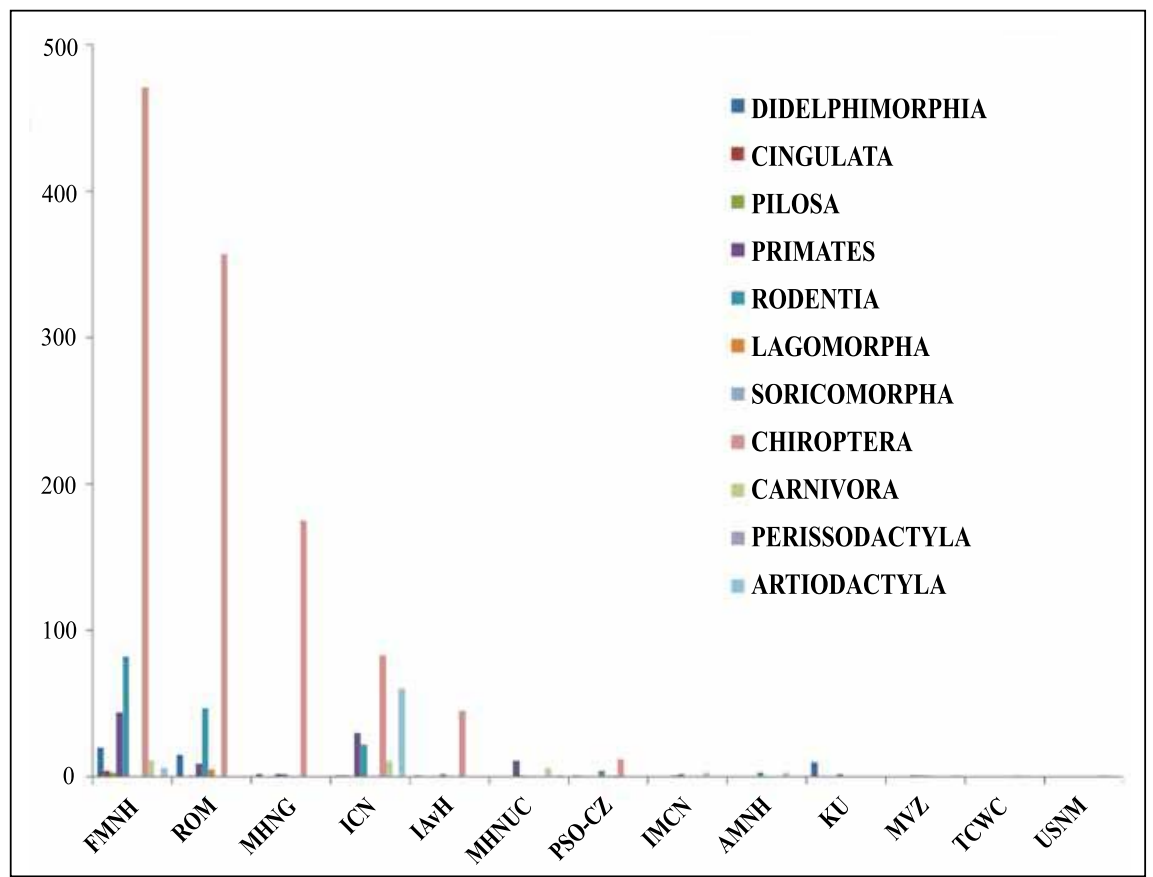

Figura 3. Ejemplares de los diferentes órdenes depositados en colecciones de referencia nacionales e internacionales procedentes del departamento de Putumayo. Los órdenes Cetacea y Sirenia no presentan ejemplares testigo. Los acrónimos de las colecciones se presentan en el texto. 
estas especies. Otra información encontrada en literatura constituye registros aislados (Hernández-Camacho, 1955; Hershkovitz, 1963; Hernández-Camacho \& BarrigaBonilla, 1966; Aellen, 1970; Marinkelle \& Cadena, 1972; Hershkovitz, 1997; Lim \& Emgstron, 2001; Riascos, 2001; Velazco, 2005; Rivas-Pava et al., 2007; Gardner, 2008; Velazco \& Gardner, 2009; Noguera-Urbano \& Montenegro, 2011, entre otros) y registros generales para el departamento (Cuervo et al., 1986; Tamsitt et al., 1986, Rodríguez-Mahecha et al., 1995, 2006a; Alberico et al., 2000; Mantilla-Meluk et al., 2009).

Stevenson et al., (2006) mencionaron que hay una tesis sobre Artiodactyla realizada en el departamento de Putumayo y un estudio relacionado con la danta Tapirus terrestris (Perissodactyla), no fue posible localizar dichas fuentes. Desconocemos de trabajos sistemáticos de inventario adicionales al previamente mencionado realizados en las últimas décadas. La cantidad de ejemplares testigo generada en los últimos 10 años es inferior a 50 individuos.

\section{Riqueza de especies y distribución}

Registramos un total de 154 especies de mamíferos para el departamento de Putumayo, pertenecientes a 108 géneros, 37 familias y 13 órdenes. Los órdenes más ricos son Chiroptera (66 especies), Rodentia (24), Carnivora (19) y Primates (15) (Figura 4). Según Rodríguez-Mahecha et al., (2006a) y la IUCN (2012), 19 especies (Tabla 1) se encuentran en riesgo de amenaza de extinción, con mayor número de especies de los órdenes Carnivora y Primates.

De los cinco distritos biogeográficos presentes en el departamento sólo tres cuentan con registros de mamíferos (Figura 1a). El distrito Alto Putumayo es el que presentan el mayor número de registros (236) y especies (136) (Tabla 2); mientras que para el Distrito Bosques Andinos Nariño Oriental, se cuenta tan solo con cinco registros de cinco especies.

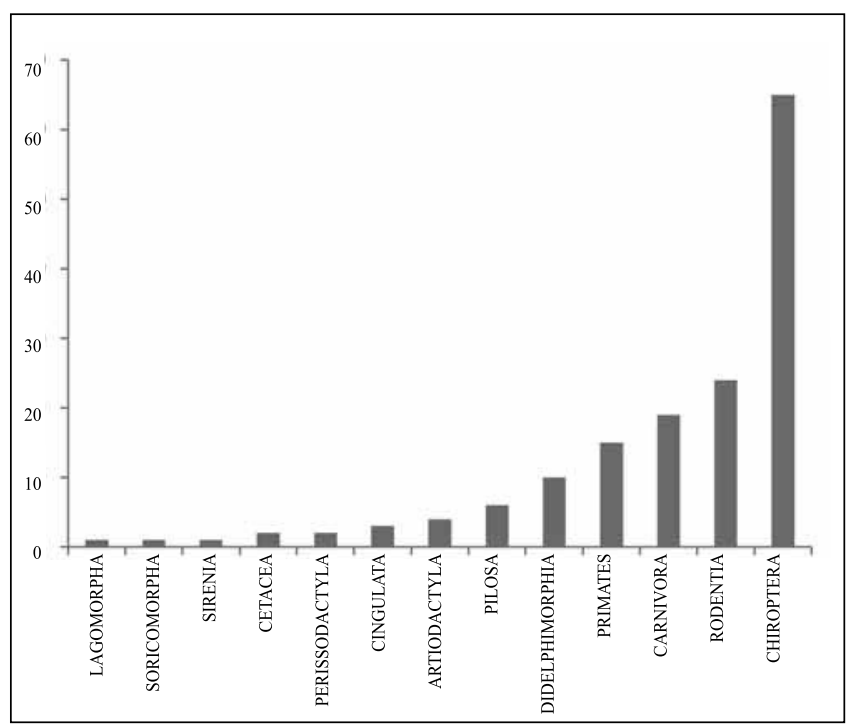

Figura 4. Número de especies por orden registradas en el departamento de Putumayo.

Para las divisiones de Olson et al., (2001) (Figura 1b), los registros provienen de dos de las cinco eco-regiones propuestas: la selva húmeda del Napo con 131 especies (262 registros) y el Bosque Montaño de la Cordillera Oriental con 49 especies (59 registros) (Tabla 2 ).

Los municipios con mayor número de reporte de especies y registros fueron Puerto Leguízamo (100 especies; 133 registros), seguido por Orito (55 especies; 68 registros) y Mocoa (52 especies; 69 registros) (ver Tabla 3).

El mayor número de especies amenazadas se encuentra en el Distrito Alto Putumayo (16 especies) seguido del Distrito Kofán (5) mientras que para las eco-regiones, el mayor número se encuentra en la Selva o bosque húmedo del Napo con 16 especies (Tabla 2).

Tabla 2. Número de especies y registros por unidad de regionalización

\begin{tabular}{|l|l|c|c|c|}
\hline \multirow{2}{*}{$\begin{array}{c}\text { Tipo de } \\
\text { Regionalización }\end{array}$} & \multicolumn{1}{|c|}{ Unidad } & $\begin{array}{c}\text { Número de espe- } \\
\text { cies por unidad }\end{array}$ & $\begin{array}{c}\text { Número de } \\
\text { registros por } \\
\text { Unidad }\end{array}$ & $\begin{array}{c}\text { Número de } \\
\text { especies } \\
\text { amenazadas }\end{array}$ \\
\hline \multirow{2}{*}{ Tipo de eco-región } & Bosque montano de la cordillera Oriental & 49 & 59 & 7 \\
\cline { 2 - 5 } & Selva o bosque húmedo del Napo & 131 & 262 & 16 \\
\hline \multirow{2}{*}{ Distritos Biogeográficos } & Distrito Alto Putumayo & 136 & 236 & 16 \\
\cline { 2 - 6 } & Distrito bosques andinos Nariño Oriental & 5 & 5 & 3 \\
\cline { 2 - 6 } & Distrito Kofán & 61 & 80 & 5 \\
\hline Localidad imprecisa & & & & \\
\hline
\end{tabular}


Tabla 3. Número de especies, especies amenazadas y registros por municipio. Los municipios de Puerto Caicedo, Puerto Guzmán, San Francisco, Santiago y Colón no presentaron registros

\begin{tabular}{|l|c|c|c|}
\hline Municipio & Número de especies & Número de registros & $\begin{array}{c}\text { Número de } \\
\text { especies } \\
\text { amenazadas }\end{array}$ \\
\hline Puerto Leguízamo & 100 & 133 & 14 \\
\hline Orito & 55 & 68 & 4 \\
\hline Mocoa & 52 & 69 & 7 \\
\hline Puerto Asís & 25 & 26 & 2 \\
\hline Villa Garzón & 11 & 12 & 3 \\
\hline San Miguel & 6 & 6 & 0 \\
\hline Sibundoy & 5 & 5 & 3 \\
\hline Valle del Guamués & 2 & 2 & 1 \\
\hline
\end{tabular}

\section{Lista anotada}

Presentamos a continuación las especies de mamíferos registradas para el departamento, así como las localidades, distrito biogeográfico y eco-región de la cual proceden los registros. No incluimos las siguientes especies mencionadas previamente en literatura como presentes en el departamento por constituir registros erróneos: el primate Saguinus labiatus, la cual fue mencionada por Rivas-Pava et al., (2007) como presente en el Putumayo, sin localidad precisa a partir de un ejemplar depositado en el MHNUC (MHNUC-M00030). El ejemplar corresponde a un cráneo y esqueleto completo, sin piel asociada, por lo que es difícil determinar a qué especie del género Saguinus corresponde. Descartamos su presencia en el departamento ya que $S$. labiatus se encuentra distribuida en el occidente de Brasil, oriente de Perú y Bolivia (Groves, 2005) y porque no ha sido incluida como representante de la fauna de primates de Colombia en tratados recientes (Defler, 2010).

Tampoco incluimos al murciélago Eptesicus diminutus como representante de la fauna del departamento de Putumayo (contra Rodríguez-Mahecha et al., 1995 y Polanco-Ochoa et al., 2000, seguido por otros autores por ejemplo Alberico et al., 2000; Gardner, 2008) ya que el ejemplar en el que se hizo este registro (ICN 13741) corresponde a un individuo del género Myotis basados en la forma del cráneo y el número de premolares (3/3 versus 1/2 en Eptesicus) (Ramírez-Chaves 2008). Otras observaciones se presentan en comentarios a lo largo de la lista. Los ejemplares revisados directamente se señalan con un asterisco (*). La sigla EANU hace referencia a observaciones hechas por el segundo autor.

\section{Orden Didelphimorphia}

Familia Didelphidae

1. Caluromys lanatus (Olfers, 1818)

Localidad: MOCOA: Santa Rosa de Los Kofanes $2 \mathrm{~km}$, San Antonio del Guamués.

Distrito: Alto Putumayo.

Eco-región: Selva Húmeda del Napo.

Referencias:MHNG 1118.92-1118.093.

2. Chironectes minimus (Zimmermann, 1780)

Localidad: MOCOA: Cerca a Mocoa.

Distrito: Alto Putumayo.

Eco-región: Bosques montanos de la Cordillera Oriental.

Referencias: ROM 46428-46429

3. Didelphis marsupialis Linnaeus, 1758

Localidad: MOCOA: Río Pepino. ORITO: Misión Evangelista; río Caldero.

Distrito: Alto Putumayo; Kofán.

Eco-región: Selva Húmeda del Napo.

Referencias: KU 123977-85.

4. Glironia venusta Thomas, 1912

Localidad: PUERTO LEGUÍZAMO: quebrada El Hacha.

Distrito: Alto Putumayo.

Eco-región: Selva Húmeda del Napo.

Referencias: Cuervo-Díaz et al., 1986; Rodríguez-Mahecha et al., 1995; Alberico et al., 2000.

5. Marmosa rubra Tate, 1931

Localidad: PUERTO LEGUÍZAMO: Río Mecaya.

Distrito:Alto Putumayo. 
Eco-región: Selva Húmeda del Napo.

Referencias: Rossi et al., 2010 (FMNH 70969-70977).

6. Marmosops noctivagus (Tschudi, 1845).

Localidad: MOCOA: Río Pepino. PUERTO LEGUízAMO: Río Mecaya.

Distrito: Alto Putumayo.

Eco-región: Selva Húmeda del Napo.

Referencias: ICN 14465*, 14466*, PSO-CZ*; FMNH 70953-70955, 70961; Díaz-N, 2012.

Comentarios: Polanco-Ochoa et al., 2000, registraron ejemplares depositados en el ICN (ICN 14465, 14466) como Marmosa murina, estos ejemplares fueron re-identificados como M. noctivagus por Díaz-N (2012).

7. Metachirus nudicaudatus É. Geoffroy Saint Hilaire, 1803 Localidad: ORITO: Río Caldero. PUERTO LEGUÍZAMO: Río Mecaya.

Distrito: Alto Putumayo; Kofán.

Eco-región: Selva Húmeda del Napo.

Referencias: FMNH 70956. EANU, 2008: Observación personal.

8. Micoureus demerarae (Thomas, 1905)

Localidad: PUERTO LEGUÍZAMO: Parque Nacional Natural La Paya.

Distrito: Alto Putumayo.

Eco-región: Selva Húmeda del Napo.

Referencias: Polanco-Ochoa et al., 2000 (ICN 14467)

9. Micoureus regina (Thomas, 1898)

Localidad: PUERTO LEGUÍZZAMO: Río Mecaya.

Distrito: Alto Putumayo.

Eco-región: Selva Húmeda del Napo.

Referencias: FMNH 70962, 70968.

10. Philander andersoni (Osgood, 1913)

Localidad: PUERTO ASÍS: $12 \mathrm{~km} \mathrm{~N}$ de Puerto Asís. PUERTO LEGUÍZAMO: Parque Nacional Natural La Paya.

Distrito: Alto Putumayo; Kofán.

Eco-región: Selva Húmeda del Napo.

Referencias: Rodríguez-Mahecha et al., 1995; Hershkovitz, 1997 (FMNH 70986-70987), Polanco-Ochoa et al., 2000 (ICN 21152*); Alberico et al., 2000; Lew et al., 2006 (KU 123950); Gardner, 2008.

\section{ORDEN CINGULATA}

Familia Dasypodidae

11. Cabassous unicinctus (Linnaeus, 1758)

Localidad: PUERTO LEGUíZAMO: Parque Nacional Natural La Paya.

Distrito: Alto Putumayo.
Eco-región: Selva Húmeda del Napo.

Referencia: Polanco-Ochoa et al., 2000.

12. Dasypus kappleri Krauss, 1862

Localidad: PUERTO LEGUÍZAMO: Parque Nacional Natural La Paya.

Distrito: Alto Putumayo.

Eco-región: Selva Húmeda del Napo.

Referencias: Rodríguez-Mahecha et al., 1995; PolancoOchoa et al., 2000.

13. Dasypus novemcinctus Linnaeus, 1758

Localidad: MOCOA: Río Pepino. PUERTO ASÍS: Bajo Río Guamués. PUERTO LEGUÍZAMO:Parque Nacional Natural La Paya.

Distrito: Alto Putumayo.

Eco-región: Selva Húmeda del Napo.

Referencias: Rodríguez-Mahecha et al., 1995; PolancoOchoa et al., 2000.

\section{ORDEN PILOSA}

Familia Bradypodidae

14. Bradypus variegatus $\operatorname{Schinz}, 1825$

Localidad: ORITO: Río Caldero. PUERTO LEGUíZAMO: Caño Caucaya Limoncocha. Puerto Leguízamo. Río Mecaya. Finca Velásquez, entrando por Limonconcho NW de Leguizamo.

Distrito: Alto Putumayo; Kofán.

Eco-región: Selva Húmeda del Napo.

Referencias: IAvH 590; Anderson \& Handley, 2001 (FMNH 70812-70813).

15.Choloepus didactylus (Linnaeus, 1758).

Localidad:MOCOA: Río Pepino. PUERTO LEGUízAMO: Parque Nacional Natural La Paya.

Distrito: Alto Putumayo.

Eco-región: Selva Húmeda del Napo.

Referencias: Noguera-Urbano, observación personal, año 2010.

16. Choloepus hoffmanni Peters, 1858

Localidad: PUERTO LEGUíZAMO: Parque Nacional Natural La Paya.

Distrito: Alto Putumayo.

Eco-región: Selva Húmeda del Napo.

Referencia: Polanco-Ochoa et al., 2000.

Familia Myrmecophagidae

17. Tamandua tetradactyla (Linnaeus, 1758)

Localidad: PUERTO LEGUíZAMO: Parque Nacional Natural La Paya.

Distrito: Alto Putumayo.

Eco-región: Selva Húmeda del Napo. 
Referencias: FMNH70815. Polanco-Ochoa et al., 2000.

18. Myrmecophaga tridactyla Linnaeus, 1758 Localidad: MOCOA: cerca a Mocoa. PUERTO LEGUÍZAMO: Parque Nacional Natural La Paya.

Distrito: Alto Putumayo.

Eco-región: Bosques montanos de la Cordillera Oriental, Selva Húmeda del Napo.

Referencias: ROM 63215. Rodríguez-Mahecha et al., 1995; Polanco-Ochoa et al., 2000, 2006.

\section{Familia Cyclopedidae}

19. Cyclopes didactylus (Linnaeus, 1758)

Localidad: PUERTO LEGUíZAMO: Parque Nacional Natural La Paya.

Distrito: Alto Putumayo.

Eco-región: Selva Húmeda del Napo.

Referencias: Rodríguez-Mahecha et al., 1995; PolancoOchoa et al., 2000.

\section{ORDEN PRIMATES}

\section{Familia Aotidae}

20. Aotus vociferans (Spix, 1823)

Localidad:PUERTO LEGUÍZAMO: Parque Nacional Natural La Paya. Río Mecaya

Distrito: Alto Putumayo.

Eco-región: Selva Húmeda del Napo.

Referencias: FMNH 70688-70690. Polanco-Ochoa et al., 2000; Defler, 2004; Rivas-Pava et al., 2007 (MHNUCM034*).

\section{Familia Atelidae}

21. Alouatta seniculus (Linnaeus, 1766)

Localidad: ORITO: El Tigre. Río Caldero. PUERTO LEGUÍZAMO: Parque Nacional Natural La Paya.

Distrito: Alto Putumayo; Kofán.

Eco-región: Selva Húmeda del Napo.

Referencias: PSO-CZ*. Rodríguez-Mahecha et al., 1995; Polanco-Ochoa et al., 2000. Observaciones: EANU 2008 observó una tropa 6 individuos en Orito Putumayo; a partir del seguimiento realizado a la tropa se recuperó en el suelo restos óseos de un ejemplar macho adulto. El ejemplar se encuentra en la colección PSO-CZ.

22. Ateles belzebuth É. Geoffroy Saint Hilaire, 1806 Localidad: PUERTO ASÍS: Puerto Asís. PUERTO LEGUÍZAMO: Parque Nacional Natural La Paya.

Distrito: Alto Putumayo; Kofán.

Eco-región: Selva Húmeda del Napo.
Referencias: MHNUC-M00112*. Rodríguez-Mahecha et al., 1995; Polanco-Ochoa et al., 2000; Defler, 2004; Palacios et al., 2006.

Comentario: Registrado como Ateles hybridus por RivasPava et al., 2007 (MHNUC-M00112*).

23. Lagothrix lagothricha Humboldt, 1812

Localidad: PUERTO LEGUízAMO: Parque Nacional Natural La Paya. Río Mecaya.

Distrito: Alto Putumayo.

Eco-región: Selva Húmeda del Napo.

Referencias: FMNH 70590-70600; MHNG 1076.094-1076.

Polanco-Ochoa et al., 2000.

\section{Familia Callitrichidae}

24. Callimico goeldii (Thomas, 1904)

Localidad: PUERTO LEGUÍZAMO: Parque Nacional Natural La Paya.

Distrito: Alto Putumayo.

Eco-región: Selva Húmeda del Napo.

Referencias: Hernández-Camacho \& Barriga-Bonilla, 1966 (ICN 84*); Cuervo-Díaz et al., 1986; Polanco-Ochoa et al., 2000; Alberico et al., 2000; Defler \& Rodríguez-M., 2006; Defler, 2010.

Comentarios: El primer registro de esta especie para el país (Hernández-Camacho \& Barriga-Bonilla, 1966) se realizó a partir de un ejemplar recolectado en la quebrada El Hacha en 1960 por Humberto Granados y Hugo Arévalo.

25.Callithrix (Cebuella) pygmaea Spix, 1823

Localidad: ORITO: El Tigre. Río Caldero. PUERTO ASÍS: Puerto Asís. PUERTO LEGUÍZAMO: Parque Nacional Natural La Paya. Puerto Leguízamo. SAN MIGUEL: Puerto Ospina.

Distrito: Alto Putumayo; Kofán.

Eco-región: Selva Húmeda del Napo.

Referencias: ICN 2946; INCIVA; ROM 40417-40418; MVZ 124107. Rodríguez-Mahecha et al., 1995; PolancoOchoa et al., 2000; Riascos, 2001; Defler, 2004, 2010.

Observaciones: en el bajo Putumayo (Orito, Puerto Leguízamo y Puerto Asís), individuos de esta especie son capturados para ser vendidos como mascotas en regiones vecinas como Nariño.

26. Saguinus fuscicollis (Spix, 1823)

Localidad: PUERTO LEGUÍZAMO: Parque Nacional Natural La Paya. Puerto Leguízamo.

Distrito: Alto Putumayo.

Eco-región: Selva Húmeda del Napo. 
Referencias: Polanco-Ochoa et al., 2000; Alberico et al., 2000; Rivas-Pava et al., 2007 (MHNUC-M 23E*); Defler, 2010.

Comentarios: Defler (2010) mencionó que la subespecie presente en el Putumayo es $S$. f. fuscus (Lesson, 1840), la cual puede tratarse de una especie plena a según datos moleculares (Cropp et al., 1999).

27. Saguinus graellsi (Jiménez de la Espada, 1870) Localidad: Imprecisa: Orilla izquierda del río Putumayo Distrito: No establecida por falta de localidad precisa.

Eco-región:Selva Húmeda del Napo.

Referencias: ICN.

Comentarios: Defler (2010) mencionó que la especie es común a lo largo de la carretera entre Mocoa y Puerto Asís.

28. Saguinus nigricollis (Spix, 1823)

Localidad: ORITO: Río Caldero. PUERTO LEGUÍZAMO: Puerto Leguízamo. VILLA GARZÓN: Puerto Umbría. Santa Rosa.

Distrito: Alto Putumayo; Kofán.

Eco-región: Selva Húmeda del Napo.

Referencias: Defler, 2004, 2010. EANU, 2008: Observación personal.

\section{Familia Cebidae}

29. Cebus albifrons (Humboldt, 1812)

Localidad: ORITO: El Tigre. Río Caldero. PUERTO ASÍ́S: Puerto Asís

Distrito: Kofán.

Eco-región: Selva Húmeda del Napo.

Referencias: Rodríguez-Mahecha et al., 1995; Alberico et al., 2000; Polanco-Ochoa et al., 2000; Defler, 2004, 2010.

30. Cebus apella (Linnaeus, 1758)

Localidad: PUERTO LEGUíZAMO: Parque Nacional Natural La Paya. Río Mecaya.

Distrito: Alto Putumayo.

Eco-región: Selva Húmeda del Napo.

Referencias: FMNH 70628-70631. Alberico et al., 2000; Polanco-Ochoa et al., 2000; Defler, 2004, 2010; Rivas-Pava et al., 2007 (MHNUC-M028 E*).

31. Saimiri sciureus (Linnaeus, 1758)

Localidad: ORITO: Río Caldero. PUERTO LEGUíZAMO: Parque Nacional Natural La Paya.

Distrito: Alto Putumayo; Kofán.

Eco-región: Selva Húmeda del Napo.

Referencias: FMNH 70668-70671. Rodríguez-Mahecha et al., 1995; Defler, 2004; Rivas-Pava et al., 2007 (MHNUCM00031*, M00032E*, M00033*).

\section{Familia Pitheciidae}

32. Callicebus discolor (I. Geoffroy \& Deville, 1848)

Localidad: Localidades imprecisas: Banco sur del Río Guamués. Río Putumayo. VALLE DEL GUAMUÉS: Rio Guamués.

Distrito: Alto Putumayo.

Eco-región: Selva Húmeda del Napo.

Referencias: Hershkovitz, 1990; Groves, 2005; Rodríguez et al., 2006; Defler, 2010.

33. Callicebus medemi Hershkovitz, 1963

Localidad: PUERTO LEGUÍZAMO: Río Mecaya. VILLA GARZÓN: Puerto Umbría. Localidad imprecisa: banco derecho del río Caquetá.

Distrito: Alto Putumayo.

Eco-región: Selva Húmeda del Napo.

Referencias: Hershkovitz, 1963 (FMNH 70699, Holotipo; FMNH 70692-70700, FMNH 84551); Hershkovitz, 1990; Groves, 2005.

Comentarios: La localidad tipo de esta especie es Putumayo, banco derecho del Río Caquetá, Rio Mecaya. La distribución de esta especie se restringe a Colombia, entre los ríos Caquetá y Putumayo (Hershkovitz, 1990; Veiga \& Palacios, 2008).

34. Pithecia monachus (É. Geoffroy Sant-Hilaire, 1812)

Localidad: MOCOA: Río Guamués. Río Pepino. ORITO:

Río Caldero. PUERTO LEGUíZAMO: Parque Nacional Natural La Paya. Puerto Leguízamo. Río Mecaya. VILLA GARZON: Inspección de Policía Villa Garzón.

Distrito: Alto Putumayo; Kofán.

Eco-región: Bosques montanos de la Cordillera Oriental, Selva Húmeda del Napo.

Referencias: ICN 9911, 14777. Rodríguez-Mahecha et al., 1995; Polanco-Ochoa et al., 2000; Defler, 2010; Defler et al., 2006.

\section{ORDEN RODENTIA}

Familia Sciuridae

35. Microsciurus flaviventer (Gray, 1867)

Localidad: MOCOA: Río Pepino. ORITO: Río Caldero. PUERTO LEGUÍZAMO: Río Mecaya.

Distrito: Alto Putumayo; Kofán.

Eco-región: Selva Húmeda del Napo.

Referencias: INCIVA; FMNH 71119-71121; PSO-CZ. Riascos, 2001; Rivas-Pava et al., 2007 (MHNUC-M0240).

36. Sciurus granatensis Humboldt, 1811

Localidad: MOCOA: Río Pepino. Localidad imprecisa: El Horno. 
Distrito: Alto Putumayo.

Eco-región: Selva Húmeda del Napo.

Referencias: ICN 9968.

37. Sciurus pucheranii (Fitzinger, 1867)

Localidad: MOCOA: Mocoa. Guacayaco. SAN MIGUEL:

San Miguel.

Distrito: Alto Putumayo; Kofán.

Eco-región: Bosques montanos de la Cordillera Oriental, Selva Húmeda del Napo.

Referencias: ROM 40391, 40393, 46423-46427.

38. Sciurus igniventris Wagner, 1842

Localidad: PUERTO LEGUÍZAMO: Parque Nacional Natural La Paya. Río Mecaya.

Distrito: Alto Putumayo.

Eco-región: Selva Húmeda del Napo.

Referencias: FMNH 71052, 71056. Polanco-Ochoa et al., 2000 registraron observaciones de Sciurus cf. igniventris para el PNN La Paya, pero debido a que no hay ejemplares testigo de dichas observaciones, no podemos establecer si se trata precisamente de esta especie.

39. Sciurus spadiceus Olfers, 1818

Localidad: ORITO: Río Caldero. PUERTO LEGUÍZAMO: Río Mecaya.

Distrito: Alto Putumayo; Kofán.

Eco-región: Selva Húmeda del Napo.

Referencias: FMNH 71055; PSO-CZ.

\section{Familia Cricetidae}

40. Euryoryzomys macconnellii (Thomas, 1910)

Localidad: PUERTO LEGUÍZAMO: Parque Nacional Natural La Paya. Río Mecaya.

Distrito: Alto Putumayo.

Eco-región: Selva Húmeda del Napo.

Referencias: FMNH 72041, 72060-72061. Polanco-Ochoa et al., 2000 (ICN 14468-14472).

41. Hylaeamys yunganus (Thomas, 1902)

Localidad: PUERTO LEGUÍZAMO: Río Mecaya.

Distrito: Alto Putumayo.

Eco-región: Selva Húmeda del Napo.

Referencias: FMNH 72067.

42. Neacomys spinosus (Thomas, 1882)

Localidad: ORITO: Río Caldero. PUERTO LEGUÍZA-

MO: Río Mecaya.

Distrito: Alto Putumayo; Kofán.

Eco-región:Selva Húmeda del Napo.

Referencias: MHNG 1268,054, PSO-CZ. Steppan, 1995

(FMNH 71784-71789, 71792).
43. Nectomys sp.

Localidad: MOCOA: Río Guamués. PUERTO LEGUÍ-

ZAMO: Río Mecaya.

Distrito: Alto Putu mayo.

Eco-región: Bosques montanos de la Cordillera Oriental, Selva Húmeda del Napo.

Referencias: ICN 1555*; ROM 40389, 41503; FMNH 71655-71657.

44. Oecomys bicolor (Tomes, 1860)

Localidad: MOCOA: Margen derecha del río Caquetá. SAN MIGUEL: San Miguel.

Distrito: Kofán.

Eco-región: Bosques montanos de la Cordillera Oriental, Selva Húmeda del Napo.

Referencias: ROM 40403-40404, 40425-40426

45. Oecomys concolor (Wagner, 1845)

Localidad: MOCOA: Margen derecha del río Caquetá. Mocoa.

Distrito: Alto Putumayo.

Eco-región: Bosques montanos de la Cordillera Oriental.

Referencias: ROM 40394-40397, 40400-40401, 4150141502.

46. Oecomys superans Thomas, 1911

Localidad: PUERTO LEGUÍZAMO: Río Mecaya.

Distrito: Alto Putumayo.

Eco-región:Selva Húmeda del Napo.

Referencias: FMNH 72017-72021; 72023, 72027-72029;

72031, 72033-72034, 72042, 72090. Alberico et al., 2000.

47. Reithrodontomys mexicanus (Saussure, 1860).

Localidad: Localidad imprecisa.

Distrito: No establecida por falta de localidad precisa.

Eco-región: No establecida por falta de localidad precisa.

Referencia: Alberico et al., 2000.

48. Thomasomys cinereiventer J.A. Allen, 1912

Localidad: Localidad imprecisa.

Distrito: No establecida por falta de localidad precisa.

Eco-región: No establecida por falta de localidad precisa.

Referencias: Alberico et al., 2000.

49. Transandinomys talamancae (Allen, 1891)

Localidad: Localidad imprecisa.

Distrito: No establecida por falta de localidad precisa.

Eco-región: No establecida por falta de localidad precisa.

Referencia: Alberico et al., 2000.

Familia Echimyidae

50. Mesomys hispidus (Desmarest, 1817) 
Localidad: PUERTO LEGUÍZAMO: Río Mecaya.

Distrito: Alto Putumayo.

Eco-región: Selva Húmeda del Napo.

Referencias: FMNH 71125-71127.

\section{Proechimys simonsi Thomas, 1900}

Localidad:PUERTO LEGUÍZAMO: Río Mecaya.

Distrito: Alto Putumayo.

Eco-región:Selva Húmeda del Napo.

Referencias: FMNH 71148-71156.

Comentarios: Polanco-Ochoa et al., 2000, registraron ejemplares de Proechimys sp. para el PNN La Paya, pero desafortunadamente no hay ejemplares testigo para corroborar su identidad taxonómica.

\section{Familia Erethizontidae.}

\section{Coendou sp.}

Localidad: MOCOA: Serranía El Churumbelo.

Distrito: Distrito Alto Putumayo.

Eco-región:Bosques montanos de la Cordillera Oriental.

Referencia:ICN 21151.

Comentarios: No es posible identificar la especie porque se trata de un individuo inmaduro (feto).

Familia Dinomyidae.

\section{Dinomys branickii Peters, 1873}

Localidad: SIBUNDOY: Valle de Sibundoy.

Distrito: Bosques Andinos Nariño Oriental.

Eco-región:Bosques montanos de la Cordillera Oriental.

Referencias: ICN 1036-37.

\section{Familia Caviidae}

54. Cavia porcellus (Linnaeus, 1758)

Localidad: SAN MIGUEL: San Miguel. SIBUNDOY: Valle de Sibundoy.

Distrito: ver comentarios.

Eco-región:ver comentarios.

Referencias: ROM 40420.

Comentarios: Especie domesticada y mantenida en la zona Andina desde la época precolombina (Ramírez-Chaves et al., 2011).

55. Hydrochoerus hydrochaeris (Linnaeus, 1766)

Localidad: PUERTO LEGUÍZAMO:Parque Nacional Natural La Paya. VILLA GARZÓN: Río Liacaé.

Distrito: Alto Putumayo.

Eco-región: Selva Húmeda del Napo.

Referencias: MHNG 1078-03. Polanco-Ochoa et al., 2000.
Familia Dasyproctidae

56. Dasyprocta fuliginosa Wagler, 1832

Localidad: ORITO: Río Caldero. PUERTO ASÍS: 17 km $\mathrm{N}$ de Puerto Asís. PUERTO LEGUÍZAMO:Parque Nacional Natural La Paya. Río Mecaya.

Distrito: Alto Putumayo; Kofán.

Eco-región: Selva Húmeda del Napo.

Referencias: INCIVA; PSO-CZ; FMNH 70796-70978. Rodríguez-Mahecha et al., 1995; Polanco-Ochoa et al., 2000; Riascos, 2001.

Comentarios: en Orito esta especie es cazada por su carne principalmente para consumo familiar.

57. Myoprocta pratti Pocock, 1913

Localidad: ORITO: Río Caldero. PUERTO LEGUÍZAMO: Río Mecaya.

Distrito: Alto Putumayo; Kofán.

Eco-región: Selva Húmeda del Napo.

Referencias: FMNH 71133-71134.

Comentarios: un ejemplar con procedencia de Puerto Leguízamo, objeto de decomiso a cazadores fue observado en Mocoa.

\section{Familia Cuniculidae}

58. Cuniculus paca (Linnaeus, 1766)

Localidad: MOCOA: Margen derecha del Río Caquetá, 2 $\mathrm{Km}$. al occidente de las bocas del río Orteguaza. Mocoa, Río Pepino. ORITO: Río Caldero. PUERTO ASÍS: Bajo Río Guamués. PUERTO LEGUÍZAMO: Parque Nacional Natural La Paya. Río Mecaya.

Distrito: Alto Putumayo; Kofán.

Eco-región: Bosques montanos de la Cordillera Oriental, Selva Húmeda del Napo.

Referencias: Rodríguez-Mahecha et al., 1995; PolancoOchoa et al., 2000; EANU, 2008: Observación personal.

Comentarios: esta especie es cazada por su carne principalmente para consumo familiar. Algunos ejemplares son cuidados en el Centro Experimental Amazónico, tras su decomiso a cazadores.

\section{ORDEN LAGOMORPHA}

\section{Familia Leporidae}

59. Sylvilagus brasiliensis (Linnaeus, 1758)

Localidad: MOCOA: Mocoa. Río Pepino.

Distrito: Alto Putumayo; Kofán.

Eco-región: Bosques montanos de la Cordillera Oriental, Selva Húmeda del Napo.

Referencias: ROM 40383, 40385, 40390, 41504-05. 


\section{ORDEN SORICOMORPHA}

Familia Soricidae

\section{Cryptotis sp.}

Localidad: SIBUNDOY: Valle de Sibundoy.

Distrito: Bosques Andinos Nariño Oriental.

Eco-región: Bosques montanos de la Cordillera Oriental.

Referencias: $\mathrm{PSO}-\mathrm{CZ}^{*}$.

Comentarios:El ejemplar es una piel y un cráneo incompleto, cuyas características no posibilitan su determinación exacta hasta el momento.

\section{ORDEN CHIROPTERA}

\section{Familia Emballonuridae}

61. Cormura brevirostris (Wagner, 1843)

Localidad: SAN MIGUEL: San Miguel.

Distrito: Kofán.

Eco-región: Selva Húmeda del Napo.

Referencias: ROM 40380-82.

62. Diclidurus ingens Hernández-Camacho, 1955

Localidad: PUERTO LEGUÍZAMO: Puerto Leguízamo.

Distrito: Alto Putumayo.

Eco-región: Selva Húmeda del Napo.

Referencias: Hernández-Camacho, 1955 (ICN 546, Holotipo); Aellen, 1970; Cadena \& González-H., 2006.

Comentario: La localidad tipo de esta especie es "Puerto Leguízamo, margen izquierda del río Putumayo, ca. a la desembocadura del río Caucaya, junto al poblado de este nombre".

63. Peropteryx macrotis (Wagner, 1843)

Localidad: MOCOA: Cerca a Mocoa.

Distrito: Alto Putumayo.

Eco-región: Bosques montanos de la Cordillera Oriental.

Referencias: ROM 41472-78, 41480-94, 41496-41500, 62262-65, 62268-69, 63244-45; MHNG 1902.076*, MHNG1902.077, MHNG 1902.078*, MHNG 1902.079*, MHNG 1902.080*, MHNG 1902.081*, MHNG 1902.082*.

64. Rhynchonycteris naso (Wied-Neuwied, 1820)

Localidad: MOCOA: San Antonio. PUERTO LEGUÍZAMO: Parque Nacional Natural La Paya. Vereda El Guadual, finca de Pablo Aguirre, Loma 1. Hacienda Hernández Río Caucaya, Lomas II.

Distrito: Alto Putumayo.

Eco-región: Selva Húmeda del Napo.

Referencias: MNHG 1907.046-1907.047-1907.048. Polanco-Ochoa et al., 2000 (ICN 13761-62).

65. Saccopteryx bilineata (Temminck, 1838)

Localidad: MOCOA: Cascada Canalendres, vereda el Zarzal. Mocoa. ORITO: Orito I, Campamento Texaco.
PUERTO ASÍS: Vereda Cocaya. VILLA GARZÓN: Puerto Limón.

Distrito: Alto Putumayo.

Eco-región: Bosques montanos de la Cordillera Oriental. Selva Húmeda del Napo.

Referencias: MHNG 1902.064*, MHNG 1902.065*, MHNG 1902.066*, MHNG 1902.067*, MHNG 1902.068*, MHNG 1902.069*, MHNG 1923.065*, MHNG 1923.066*, MHNG 1923.067*, MHNG 1923.068*, MHNG 1923.069*, MHNG 1923.070*, MHNG 1923.071*, MHNG 1923.072*. ROM 40369, 40371, 46350-52, 49185-87, 63243.

66. Saccopteryx leptura (Schreber, 1774)

Distrito: PUERTO LEGUÍZAMO: Río Mecaya.

Distrito: Alto Putumayo.

Eco-región: Selva Húmeda del Napo.

Referencias: FMNH 72354-72357, 113902.

Familia Noctilionidae

67. Noctilio albiventris Desmarest, 1818

Localidad: MOCOA: San Antonio. ORITO: Orito. PUERTO LEGUÍZAMO: Parque Nacional Natural La Paya. Río Mecaya. Unidad vega río Caucayá.

Distrito: Alto Putumayo; Kofán.

Eco-región: Selva Húmeda del Napo.

Referencias: MNHG 1905.060*, MNHG 1907.025*; FMNH 71528. Polanco-Ochoa et al., 2000.

\section{Familia Molossidae}

68. Cynomops greenhalli Goodwin, 1958

Localidad: PUERTO LEGUÍZAMO: Puerto Leguízamo.

Distrito: Alto Putumayo.

Eco-región:Selva Húmeda del Napo.

Referencias: Marinkelle \& Cadena, 1972 registraron dos especímenes depositados en la colección de la Universidad de Los Andes, Bogotá (MHNU 12500, 14418).

69. Cynomops paranus (O. Thomas, 1901)

Localidad: MOCOA: Mocoa.

Distrito: Alto Putumayo.

Eco-región: Bosques montanos de la Cordillera Oriental.

Referencias: Eger, 2008 (ROM 41479).

70. Molossops neglectus Williams \& Genoways, 1980

Localidad: PUERTO LEGUÍZAMO: Puerto Leguízamo a $20 \mathrm{~km} \mathrm{~S}$ de Gamarra Cesar.

Distrito: Alto Putumayo.

Eco-región:Selva Húmeda del Napo.

Rerefencia: Lim \& Engstrom, 2001 (ROM 69532). 


\section{Molossus molossus (Pallas, 1766)}

Localidad: PUERTO ASÍS: Puerto Asís. PUERTO LEGUÍZAMO: Parque Nacional Natural La Paya.

Distrito: Alto Putumayo; Kofán.

Eco-región:Selva Húmeda del Napo.

Referencias: MHNG 1899.080*, MHNG 1899.081*, MHNG 1899.082*, MHNG 1899.083*, MHNG1899.084*, MHNG 1899.085*, MHNG 1899.086*, MHNG 1899.087*, MHNG 1901.11*.MHNG 1914.065-1914.069-1914.075. Polanco-Ochoa et al., 2000 (ICN 13745-13746).

72. Molossus pretiosus Miller, 1902

Localidad: PUERTO LEGUÍZAMO: Puerto Leguízamo.

Distrito: Alto Putumayo.

Eco-región: Selva Húmeda del Napo.

Referencias: Marinkelle \& Cadena, 1972.

73. Molossus rufus É. Geoffroy Saint-Hilaire, 1805

Distrito: PUERTO LEGUÍZAMO: Río Mecaya.

Distrito: Alto Putumayo.

Eco-región: Selva Húmeda del Napo.

Referencias: FMNH 72217. Alberico et al., 2000.

Familia Phyllostomidae

Phyllostominae

74. Lophostoma brasiliense Peters 1866

Localidad: ORITO: Río Caldero.

Distrito: Kofán.

Eco-región: Selva Húmeda del Napo.

Referencias: PSO-CZ. Mantilla-Meluk et al., 2009.

75. Lophostoma silvicolum d'Orbygni 1836

Localidad: ORITO: Río Caldero. PUERTO LEGUÍZAMO: Parque Nacional Natural La Paya. Río Caucayá. Río Mecaya. Vereda El Guadual, finca de Pablo Aguirre, Loma 1. Finca Velásquez, entrando por Limonconcho NW de Leguizamo. SAN MIGUEL: Santa Rosa de Sucumbíos.

Distrito: Alto Putumayo; Kofán.

Eco-región: Selva Húmeda del Napo.

Referencias: FMNH 71529-71532, 71147-72148, 104855; PSO-CZ. Polanco-Ochoa et al., 2000 (ICN 13777-13780); Mantilla-Meluk et al., 2009.

76. Macrophyllum macrophyllum (Schinz, 1821)

Localidad: ORITO: San Antonio del Guamués.

Distrito: Kofán.

Eco-región: Selva Húmeda del Napo.

Referencias: MHNG 1907.021*.

77. Micronycteris megalotis (Gray, 1842)

Localidad: ORITO: Orito I, Estación de Bombeo Texaco. PUERTO LEGUÍZAMO: Río Mecaya.
Distrito: Alto Putumayo; Kofán.

Eco-región: Selva Húmeda del Napo.

Referencias: FMNH 72159, 72262-72264, 72374; MHNG 1908.046*.

78. Micronycteris minuta (Gervais, 1856)

Localidad: MOCOA: Mocoa. PUERTO LEGUÍZAMO: Río Mecaya. VILLA GARZÓN: Puerto Limón.

Distrito: Alto Putumayo.

Eco-región: Bosques montanos de la Cordillera Oriental. Selva Húmeda del Napo.

Referencias: FMNH 72158, 72160-72162, 72284-72286; ROM 56575; PSO-CZ.

79. Mimon crenulatum (É. Geoffroy Saint-Hilaire, 1810)

Localidad: MOCOA: Mocoa.

Distrito: Alto Putumayo.

Eco-región: Bosques montanos de la Cordillera Oriental.

Referencias: PSO-CZ.

80. Phyllostomus elongatus (E. Geoffroy St. Hilaire 1810) Localidad: MOCOA: San Antonio. PUERTO LEGUÍZAMO: Parque Nacional Natural La Paya. Vereda El Guadual, finca de Pablo Aguirre, Loma 1. Río Mecaya.

Distrito: Alto Putumayo; Kofán.

Eco-región: Selva Húmeda del Napo.

Referencias: FMNH 71919, 71527. Polanco-Ochoa et al., 2000 (ICN 13750-13752); Rodríguez-Posada \& SánchezPalomino, 2009 (ICN 13748-13752); Mantilla-Meluk $\boldsymbol{e t}$ al., 2009.

Comentarios: Dos ejemplares registrados como Phyllostomus discolor (Wagner, 1843) por Polanco-Ochoa et al., 2000 (ICN 13748*-13749*) fueron reasignados a P. elongatus por Rodríguez-Posada \& Sánchez-Palomino, 2009.

81. Phyllostomus hastatus (Pallas, 1767)

Localidades: ORITO: Orito. PUERTO ASÍS: Vereda Cocaya.

Distrito: Alto Putumayo; Kofán.

Eco-región: Selva Húmeda del Napo.

Referencias: MHNG 1906.02; ROM 49182.

82. Phylloderma stenops Peters, 1865

Localidad: PUERTO LEGUÍZAMO: Parque Nacional Natural La Paya.

Distrito: Alto Putumayo.

Eco-región: Selva Húmeda del Napo.

Referencias: FMNH 71919, 71527. Polanco-Ochoa et al., 2000 (ICN 13747).

83. Trachops cirrhosus (Spix 1823)

Localidad: PUERTO LEGUíZAMO: Parque Nacional Natural La Paya. Río Mecaya. 
Distrito: Alto Putumayo.

Eco-región: Selva Húmeda del Napo.

Referencias: FMNH 71533, 72149-72151. Polanco-Ochoa et al., 2000 (ICN 13781-13783); Mantilla-Meluk et al., 2009.

84. Tonatia saurophila Koopman \& Williams, 1951

Localidad: ORITO: Estación de bombeo Guamués.

Distrito: Kofán.

Eco-región: Bosques montanos de la Cordillera Oriental.

Referencias: Williams et al., 1995 (FMNH).

\section{Stenodermatinae}

85. Artibeus concolor Peters, 1865

Localidad: PUERTO LEGUÍZAMO: Puerto Leguízamo.

Distrito: Alto Putumayo.

Eco-región: Selva Húmeda del Napo.

Referencias: TCWC 26046.

86. Artibeus jamaicensis Leach, 1821

Localidad: PUERTO LEGUÍZAMO:Parque Nacional Natural La Paya. Río Mecaya.

Distrito: Alto Putumayo.

Eco-región: Selva Húmeda del Napo.

Referencias: Polanco-Ochoa et al., 2000 (ICN 1370913712).

87. Artibeus lituratus (Olfers, 1818)

Localidad: ORITO: Río Caldero. PUERTO LEGUÍZAMO: Base Fluvial, Puerto Leguízamo.

Distrito: Alto Putumayo; Kofán.

Eco-región: Selva Húmeda del Napo.

Referencias: ROM 49446, 49448-52, 49455-56, 49458-65, 49468-71, 49475-77, 49479, 49485, 49488, 49490, 49493, 49508-21, 49524-29, 49540, 49590, 49598-49604; PSO-CZ.

Polanco-Ochoa et al., 2000 (ICN 13713-13717).

88. Artibeus obscurus (Schinz, 1821)

Localidad: PUERTO LEGUÍZAMO: Parque Nacional Natural La Paya.

Distrito: Alto Putumayo.

Eco-región: Selva Húmeda del Napo.

Referencias: FMNH 113402, 113407-113408. PolancoOchoa et al., 2000 (ICN 13708).

89. Artibeus planirostris (Spix, 1823)

Localidad: ORITO: Estación de bombeo Guamués. Río Caldero. PUERTO LEGUÍZAMO: Parque Nacional Natural La Paya.

Distrito: Alto Putumayo; Kofán.

Eco-región: Bosques montanos de la Cordillera Oriental; Selva Húmeda del Napo.
Referencias: FMNH 113698; PSO-CZ. Polanco-Ochoa et al., 2000 (ICN 13718-13720); Alberico et al., 2000.

90. Chiroderma villosum Peters, 1860

Localidad: ORITO: Estación de bombeo Guamués. PUERTO LEGUÍZAMO: Parque Nacional Natural La Paya. VALLE DEL GUAMUÉS: Rio Guamués.

Distrito: Alto Putumayo; Kofán.

Eco-región: Bosques montanos de la Cordillera Oriental; Selva Húmeda del Napo.

Referencias: FMNH 113169, 113624. Polanco-Ochoa et al., 2000 (ICN 13739); Alberico et al., 2000.

91. Chiroderma salvini Dobson, 1878

Localidad: PUERTO LEGUÍZAMO: Parque Nacional Natural La Paya. Vereda El Guadual, finca de Pablo Aguirre, Loma 1.

Distrito: Alto Putumayo.

Eco-región: Selva Húmeda del Napo.

Referencias: Polanco-Ochoa et al., 2000 (ICN 1373513738); Alberico et al., 2000.

92. Chiroderma trinitatum Goodwin, 1958

Localidad: El Horno

Distrito: No establecida por falta de localidad precisa.

Eco-región: No establecida por falta de localidad precisa.

Referencias: ROM 63234-63238.

93. Dermanura phaeotis Miller, 1902

Localidad: MOCOA: Carretera entre Sibundoy y Mocoa, Localidad El Mirador.

Distrito: Alto Putumayo.

Eco-región: Bosques montanos de la Cordillera Oriental.

Referencias: IAvH 6835, IAvH 6843

94. Enchisthenes hartii Thomas, 1892

Localidad:El Horno. MOCOA: Guascayaco.

Distrito: Alto Putumayo.

Eco-región:Bosques montanos de la Cordillera Oriental.

Referencias: ROM 40218, 63242.

95. Mesophylla macconnelli Thomas, 1901

Localidad: El Horno. MOCOA: Guascayaco. Mocoa. San Antonio. ORITO: Estación de bombeo Guamués. Río Caldero.

Distrito: Alto Putumayo; Kofán.

Eco-región: Bosques montanos de la Cordillera Oriental; Selva Húmeda del Napo.

Referencias: ROM 49213-14, 56577-78, 56580-88, 6324662; PSO-CZ; FMNH 113367, 113630, 113640, 113904113906. 
96. Platyrrhinus brachycephalus (Rouk \& Carter, 1972) Localidad: MOCOA: San Antonio. ORITO: Estación de bombeo Guamués.

Distrito: Alto Putumayo; Kofán.

Eco-región: Bosques montanos de la Cordillera Oriental; Selva Húmeda del Napo.

Referencias: Velazco, 2005 (FMNH 113657, 114018114022).

97. Platyrrhinus angustirostris Velazco, Gardner \& Patterson, 2010

Localidad: PUERTO LEGUíZAMO: Parque Nacional Natural La Paya.

Distrito: Alto Putumayo.

Eco-región:Selva Húmeda del Napo.

Referencias: ICN 13753-13755.

Comentarios: Estos ejemplares fueron registrados como Platyrrhinus helleri por Polanco-Ochoa et al., 2000.

98. Platyrrhinus infuscus (Peters, 1880)

Localidad: MOCOA: San Antonio. ORITO: Estación de bombeo Guamués. PUERTO LEGUÍZAMO: Río Mecaya. Distrito: Alto Putumayo; Kofán.

Eco-región: Bosques montanos de la Cordillera Oriental; Selva Húmeda del Napo.

Referencias: Polanco-Ochoa et al., 2000 (ICN 1375613757); Alberico et al., 2000; Velazco, 2005 (FMNH 72125, $72123,113413,113415,113417,113699,113752,113898-$ 113900, 114128-29); Mantilla-Meluk et al., 2009.

99. Platyrrhinus ismaeli Velazco, 2005

Localidad: MOCOA: Carretera entre Sibundoy y Mocoa, Localidad El Mirador.

Distrito: Alto Putumayo.

Eco-región: Bosques montanos de la Cordillera Oriental.

Referencias: Velazco \& Gardner, 2009 (IAvH-M 6818, IAvH 6823).

100. Platyrrhinus nigellus (Gardner \& Carter, 1972)

Localidad: MOCOA: Carretera entre Sibundoy y Mocoa, Localidad El Mirador.

Distrito: Alto Putumayo.

Eco-región: Bosques montanos de la Cordillera Oriental.

Referencias:Velazco \& Gardner, 2009 (IAvH-M 6817, IAvH 6819, IAvH 6825).

101. Uroderma bilobatum Peters, 1866

Localidad: PUERTO ASÍS: Puerto Asís. PUERTO LEGUÍZAMO: Parque Nacional Natural La Paya. Vereda El Guadual, finca de Pablo Aguirre, Loma 1.

Distrito: Alto Putumayo; Kofán.

Eco-región: Selva Húmeda del Napo.

Referencias: MHNG 1903.79. Polanco-Ochoa et al., 2000 (ICN 13784).
102. Uroderma magnirostrum Davis, 1968

Localidad: ORITO: San Antonio del Guamués.

Distrito: Kofán.

Eco-región: Selva Húmeda del Napo.

Referencia: MHNG 1907.064*.

103. Vampyressa thyone Thomas, 1909

Localidad: PUERTO LEGUÍZAMO: Parque Nacional Natural La Paya. Vereda El Guadual, finca de Pablo Aguirre, Loma 1.

Distrito: Alto Putumayo.

Eco-región:Selva Húmeda del Napo.

Referencias: ICN 13785.

Comentarios: Registrados como Vampyressa pusilla por Polanco-Ochoa et al., 2000.

104. Vampyriscus bidens Dobson, 1878

Localidad: ORITO: Estación de bombeo Guamués.

Distrito: Kofán.

Eco-región: Bosques montanos de la Cordillera Oriental.

Referencias: FMNH 113643.

105. Vampyrodes caraccioli (Thomas, 1889)

Localidad: MOCOA: San Antonio. ORITO: San Antonio del Guamués. PUERTO ASIS: Puerto Asís. PUERTO LEGUíZAMO: Puerto Leguízamo. Río Caucayá.

Distrito: Alto Putumayo; Kofán.

Eco-región: Selva Húmeda del Napo.

Referencias: IAvH-M 593; MHNG 1902.075; MHNG 1907. 007. Velazco \& Simmons, 2011 (FMNH 113948).

106. Sturnira bidens (Thomas, 1915)

Localidad: MOCOA: Carretera entre Sibundoy y Mocoa, Localidad El Mirador. PUERTO LEGUÍZAMO: La Victoria.

Distrito: Alto Putumayo.

Eco-región: Bosques montanos de la Cordillera Oriental; Selva Húmeda del Napo.

Referencias: IAvH 6839, IAvH 6845-6846, IAvH 68496850; FMNH 11302-113207. Marinkelle \& Cadena, 1972.

107. Sturnira lilium (É. Geoffroy Saint-Hilaire, 1810)

Localidad: MOCOA: San Antonio. ORITO: Orito I, Estación de Bombeo Texaco. PUERTO LEGUíZAMO: Parque Nacional Natural La Paya.

Distrito: Alto Putumayo; Kofán.

Eco-región: Selva Húmeda del Napo.

Referencias: MHNG 1905.082; MHNG 1906.65*, MHNG 1906.084-1906.085, MHNG 1906.086*, MHNG 1906.088, MHNG 1906.089*, MHNG-1907.001*, MHNG 1907.002*, MHNG 1907.003*, MHNG 1907.028*, MHNG 1907.029*, MHNG 1907.031*, MHNG 1907.032*, MHNG 1907.033*, MHNG 1907.044, MHNG 1907.062*, MHNG 1908.22*. Polanco-Ochoa et al., 2000 (ICN 13763-13771). 
108. Sturnira ludovici Anthony, 1924

Localidad:PUERTO LEGUÍZAMO: La Victoria. Parque Nacional Natural La Paya.

Distrito: Alto Putumayo.

Eco-región: Selva Húmeda del Napo.

Referencias: FMNH 113480. Polanco-Ochoa et al., 2000 (ICN 13772-13773).

109. Sturnira magna de la Torre, 1966

Localidad: ORITO: Estación de bombeo Guamués. Río Caldero.

Distrito: Kofán.

Eco-región: Bosques montanos de la Cordillera Oriental; Selva Húmeda del Napo.

Referencias: FMNH; PSO-CZ. Tamsitt et al., 1986; Alberico et al., 2000, Mantilla-Meluk et al., 2009.

110. Sturnira tildae de la Torre 1959

Localidad: ORITO: Estación de bombeo Guamués. PUERTO ASÍS: Vereda Cocaya. PUERTO LEGUÍZAMO: Parque Nacional Natural La Paya.

Distrito: Alto Putumayo; Kofán.

Eco-región: Bosques montanos de la Cordillera Oriental; Selva Húmeda del Napo.

Referencias: MHNG 1902.058*. Marinkelle \& Cadena, 1971; Polanco-Ochoa et al., 2000 (ICN 13774-13776); Mantilla-Meluk et al., 2009.

\section{Desmodontinae}

111. Desmodus rotundus (E. Geoffroy Saint-Hilaire, 1810) Localidad: MOCOA: Guascayaco. Mocoa. ORITO: Orito. Villa Garzón: Villa Garzón.

Distrito: Alto Putumayo; Kofán.

Eco-región: Bosques montanos de la Cordillera Oriental; Selva Húmeda del Napo.

Referencias: ROM 46362-46364.

112. Diaemus youngii (Jentink, 1893)

Localidad: PUERTO LEGUÍZAMO: Río Mecaya.

Distrito: Alto Putumayo.

Eco-región: Selva Húmeda del Napo.

Referencias: Lay, 1962 (FMNH 72142); Pinto et al., 2007.

\section{Glossophaginae}

113. Anoura caudifer (É. Geoffroy Saint-Hilaire, 1818) Localidad: ORITO: Estación de bombeo Guamués. PUERTO LEGUÍZAMO: La Victoria.

Distrito: Alto Putumayo; Kofán.

Eco-región: Bosques montanos de la Cordillera Oriental; Selva Húmeda del Napo.

Referencias: FMNH 11391, FMNH 113364, FMNH113502113509, FMNH 114101-114103.
114. Anoura geoffroyi Gray, 1838

Localidad: MOCOA: Carretera entre Sibundoy y Mocoa, Localidad El Mirador. ORITO: San Antonio del Guamués.

Distrito: Alto Putumayo; Kofán.

Eco-región: Bosques montanos de la Cordillera Oriental; Selva Húmeda del Napo.

Referencias: IAvH 6813-6815, IAvH 6827-6834; MHNG 1907.019.

115. Choeroniscus minor (Peters, 1868)

Localidad: PUERTO LEGUÍZAMO: Parque Nacional Natural La Paya.

Distrito: Alto Putumayo.

Eco-región: Selva Húmeda del Napo.

Referencias: Polanco-Ochoa et al., 2000 (ICN 13740).

116. Glossophaga soricina (Pallas, 1766)

Localidad: VILLA GARZÓN: Puerto Limón.

Distrito: Alto Putumayo.

Eco-región: Selva Húmeda del Napo.

Referencias: ROM 40368.

117. Lonchophylla thomasi Allen, 1904

Localidad: PUERTO LEGUÍZAMO: Parque Nacional Natural La Paya.

Distrito: Alto Putumayo.

Eco-región: Selva Húmeda del Napo.

Referencias: Polanco-Ochoa et al., 2000 (ICN 1374313744).Mantilla-Meluk et al., 2010 (ICN 13742*, 13743*, $\left.13744^{*}\right)$

\section{Carolliinae}

118. Carollia brevicauda (Schinz, 1821)

Localidad: MOCOA: Carretera entre Sibundoy y Mocoa, Localidad El Mirador.

Distrito: Alto Putumayo.

Eco-región: Bosques montanos de la Cordillera Oriental; Selva Húmeda del Napo.

Referencias: IAvH 6856. MHNG 1904.066*, MHNG 1905.083*, MHNG 1906.100*, MHNG 1907.034*, MHNG $1907.035^{*}, \mathrm{MHNG} 1907.036^{*}, \mathrm{MHNG} 1907.037^{*}, \mathrm{MHNG}$ $1907.090 *$.

119. Carollia castanea $\mathrm{H}$. Allen, 1890

Localidad: ORITO: Río Caldero. PUERTO LEGUÍZAMO: Parque Nacional Natural La Paya. Vereda El Guadual, finca de Pablo Aguirre, Loma 1.

Distrito: Alto Putumayo; Kofán.

Eco-región: Selva Húmeda del Napo.

Referencias: PSO-CZ; MHNG 1906.090*, MHNG 1906.091*, MHNG 1906.092*, MHNG 1906.093*, MHNG 1906.095*, MHNG-1906.096*, MHNG 1907.054*, MHNG1907.049, MHNG 1907.091*. Polanco-Ochoa et al., 2000 (ICN 13721-13723). 
120. Carollia perspicillata (Linnaeus, 1758)

Localidad: MOCOA: Carretera entre Sibundoy y Mocoa, Localidad El Mirador. San Antonio. ORITO: Orito I, Estación de Bombeo Texaco. PUERTO ASÍS: Puerto Asís.

Distrito: Alto Putumayo; Kofán.

Eco-región: Bosques montanos de la Cordillera Oriental; Selva Húmeda del Napo.

Referencias: PSO-CZ; IAvH 6854-6855, IAvH 6857; MHNG 1914.043-1914.056, MHNG 1900.071-1900.075; MHNG 1906.098. Polanco-Ochoa et al., 2000 (ICN 1372413734).

121. Rhinophylla fischerae Carter, 1966

Localidad: MOCOA: San Antonio. ORITO: Estación de bombeo Guamués. Río Caldero. PUERTO LEGUÍZAMO: Parque Nacional Natural La Paya.

Distrito: Alto Putumayo; Kofán.

Eco-región: Bosques montanos de la Cordillera Oriental; Selva Húmeda del Napo.

Referencias: FMNH 113362, FMNH 113366, FMNH 113368, FMNH 113371; PSO-CZ. Polanco-Ochoa et al., 2000 (ICN 13758-13759); Alberico et al., 2000.

122. Rhinophylla pumilio Peters, 1865

Localidad: ORITO: Río Caldero. San Antonio de Guamués. PUERTO LEGUÍZAMO: Parque Nacional Natural La Paya. San Miguel: La Tagua.

Distrito: Alto Putumayo; Kofán.

Eco-región: Selva Húmeda del Napo.

Referencias: MHNG 1907.085*; PSO-CZ;FMNH 113354113358; ROM 40376, ROM 40378-40379, ROM 62536.

Polanco-Ochoa et al., 2000 (ICN 13760); Mantilla-Meluk et al., 2009.

Familia Vespertilionidae

123. Eptesicus brasiliensis (Desmarest, 1820)

Localidad: PUERTO LEGUíZAMO: Puerto Leguízamo.

Distrito: Alto Putumayo.

Eco-región: Selva Húmeda del Napo.

Referencias: ROM 69581.

124. Myotis albescens (E. Geoffroy Saint-Hilaire, 1806)

Localidad: Puerto Asís: Misión Evangelista.

Distrito: Alto Putumayo.

Eco-región: Selva Húmeda del Napo.

Referencias: MHNG 1906.010*, MHNG 1906.011*, MHNG 1906.012*.

125. Myotis nigricans (Schinz, 1821)

Localidad: MOCOA: Carretera entre Sibundoy y Mocoa, Localidad El Mirador. Mocoa. San Antonio.
Distrito: Alto Putumayo; Kofán.

Eco-región: Bosques montanos de la Cordillera Oriental; Selva Húmeda del Napo.

Referencias: IAvH 6852-6853; FMNH 113950.

126. Myotis simus Thomas, 1901

Localidad: MOCOA: Mocoa. Río Pepino.

Distrito: Kofán.

Eco-región: Bosques montanos de la Cordillera Oriental; Selva Húmeda del Napo.

Referencias: ROM 41495; PSO-CZ. Marinkelle \& Cadena, 1972; Alberico et al., 2000.

\section{ORDEN CARNIVORA}

\section{Familia Felidae}

127. Leopardus pardalis (Linnaeus, 1758)

Localidad:PUERTO LEGUÍZAMO:Parque Nacional Natural La Paya.

Distrito: Alto Putumayo.

Eco-región:Selva Húmeda del Napo.

Referencias: Rodríguez-Mahecha et al., 1995; PolancoOchoa et al., 2000; Rivas-Pava et al., 2007 (MHNUC 71E$\left.72 \mathrm{E}^{*}\right)$.

128. Leopardus tigrinus (Schreber, 1755)

Localidad: MOCOA: Mocoa. PUERTO LEGUÍZAMO:

Puerto Leguízamo.

Distrito: Alto Putumayo.

Eco-región: Bosques montanos de la Cordillera Oriental; Selva Húmeda del Napo.

Referencias: AMNH 149316-18. EANU, 2008: Observación personal.

129. Leopardus wiedii (Schinz, 1821)

Localidad: ORITO: Río Caldero. PUERTO LEGUíZAMO: Parque Nacional Natural La Paya. Río Mecaya.

Distrito: Alto Putumayo; Kofán.

Eco-región: Selva Húmeda del Napo.

Referencias: FMNH 70568. Polanco-Ochoa et al., 2000.

130. Panthera onca (Linnaeus, 1758)

Localidad: MOCOA: Río Pepino. ORITO: El Tigre. Río Caldero. PUERTO LEGUízAMO: Parque Nacional Natural La Paya. Río Consaya. VILLA GARZÓN: Villa Garzón. Distrito: Alto Putumayo; Kofán.

Eco-región: Selva Húmeda del Napo.

Referencias: FMNH 70566. Rodríguez-Mahecha et al., 1995; Polanco-Ochoa et al., 2000; Rivas-Pava et al., 2007 (MHNUC 123*, MHNUC 124*, MHNUC 133*). EANU, 2008: Observación personal. 
131. Puma concolor (Linnaeus, 1771)

Localidad: MOCOA: Río Pepino. PUERTO ASÍS: Puerto Asís. PUERTO LEGUízAMO: Parque Nacional Natural La Paya.

Distrito: Alto Putumayo; Kofán.

Eco-región: Selva Húmeda del Napo.

Referencias: USNM 544421. Rodríguez-Mahecha et al., 1995; Polanco-Ochoa et al., 2000; Jorgenson et al., 2006 c.

132. Puma yagouaroundi (É. Geoffroy Sant-Hilaire, 1803) Localidad: PUERTO ASÍS: Puerto Asís. PUERTO LEGUÍZAMO: Parque Nacional Natural La Paya.

Distrito: Alto Putumayo; Kofán.

Eco-región: Selva Húmeda del Napo

Referencias: INCIVA. Rodríguez-Mahecha et al., 1995;

Polanco-Ochoa et al., 2000; Riascos, 2001.

\section{Familia Canidae}

133. Atelocynus microtis (Sclater, 1883)

Localidad: PUERTO LEGUÍZAMO: Parque Nacional Natural La Paya.

Distrito: Alto Putumayo.

Eco-región: Selva Húmeda del Napo.

Referencias: Polanco-Ochoa et al., 2000.

134. Lycalopex culpaeus (Molina, 1782)

Localidad: SIBUNDOY: Valle del Sibundoy.

Distrito: Bosques Andinos Nariño Oriental.

Eco-región: Bosques montanos de la Cordillera Oriental.

Referencias: Jorgenson et al., $2006 \mathrm{~b}$.

135. Spheothos venaticus (Lund, 1842)

Localidad: ORITO: Río Caldero.

Distrito: Kofán.

Eco-región: Selva Húmeda del Napo.

Referencias: EANU, 2008: Observación personal.

\section{Familia Mustelidae}

136. Mustela frenata Lichtenstein, 1831

Localidad: SIBUNDOY: Valle del Sibundoy.

Distrito: Bosques Andinos Nariño Oriental.

Eco-región: Bosques montanos de la Cordillera Oriental.

Comentarios: Ramírez-Chaves: observación personal, septiembre 2012.

137. Eira barbara (Linnaeus, 1758)

Localidad: MOCOA: Río Pepino. PUERTO ASÍS: Puerto Asís. PUERTO LEGUÍZAMO: Parque Nacional Natural La Paya.

Distrito: Alto Putumayo; Kofán.

Eco-región:Selva Húmeda del Napo.
Referencias: Rodríguez-Mahecha et al., 1995; PolancoOchoa et al., 2000.

138. Galictis vittata (Schreber, 1776)

Localidad: MOCOA: Mocoa. ORITO: Río Caldero. PUERTO ASÍS: Puerto Asís.

Distrito: Alto Putumayo; Kofán.

Eco-región: Bosques montanos de la Cordillera Oriental; Selva Húmeda del Napo.

Referencias: INCIVA. Riascos, 2001; EANU, 2008: Observación personal.

139. Lontra longicaudis (Olfers, 1818)

Localidad: MOCOA: Río Pepino. ORITO: Río Caldero. PUERTO ASÍS: Puerto Asís. PUERTO LEGUÍZAMO: Parque Nacional Natural La Paya.

Distrito: Alto Putumayo; Kofán.

Eco-región: Selva Húmeda del Napo.

Referencias: ICN 1048. Polanco-Ochoa et al., 2000; Noguera-Urbano \& Montenegro-Muñoz, 2011.

140. Pteronura brasiliensis (Gmelin, 1788)

Localidad: PUERTO LEGUÍZAMO: Parque Nacional Natural La Paya. VILLA GARZÓN: Villa Garzón.

Distrito: Alto Putumayo.

Eco-región: Selva Húmeda del Napo.

Referencias: FMNH 70768. Polanco-Ochoa et al., 2000; Trujillo et al., 2006; Rivas-Pava et al., 2007 (MHNUCM062E*).

Familia Ursidae

141. Tremarctos ornatus (F.G. Cuvier, 1825)

Localidad: MOCOA: Río Pepino.

Distrito: Alto Putumayo.

Eco-región: Selva Húmeda del Napo.

Referencias: Jorgenson et al., 2006a.

Familia Procyonidae

142. Bassaricyon sp.

Localidad: MOCOA: Mocoa.Río Pepino.

Distrito: Alto Putumayo.

Eco-región: Bosques montanos de la Cordillera Oriental; Selva Húmeda del Napo.

Referencias: ICN 3725*.

143. Nasua nasua (Linnaeus, 1766)

Localidad: MOCOA: Mocoa. ORITO: Río Caldero. Río Orito. PUERTO LEGUÍZAMO: Parque Nacional Natural La Paya.

Distrito: Alto Putumayo; Kofán.

Eco-región: Bosques montanos de la Cordillera Oriental; Selva Húmeda del Napo. 
Referencias: INCIVA. Rodríguez-Mahecha et al., 1995; Polanco-Ochoa et al., 2000; Alberico et al., 2000; Riascos, 2001; EANU, 2008: Observación personal.

144. Potos flavus (Schreber, 1774)

Localidad: MOCOA: Río Pepino. ORITO: Estación de bombeo Guamués. PUERTO LEGUÍZAMO: Parque Nacional Natural La Paya. Río Mecaya.

Distrito: Alto Putumayo; Kofán.

Eco-región: Bosques montanos de la Cordillera Oriental; Selva Húmeda del Napo.

Referencias: ICN 9924*; FMNH 70713-70720. PolancoOchoa et al., 2000.

145. Procyon cancrivorus G. Cuvier, 1798

Localidad: VILLA GARZÓN: Villa Garzón.

Distrito: Alto Putumayo.

Eco-región: Selva Húmeda del Napo.

Referencias: PSO-CZ.

\section{ORDEN PERISSODACTYLA}

Familia Tapiridae

146. Tapirus pinchaque (Roulin, 1829)

Localidad: SIBUNDOY: Valle del Sibundoy.

Distrito: Bosques Andinos Nariño Oriental.

Eco-región: Bosques montanos de la Cordillera Oriental.

Referencia: Lizcano et al., 2006.

147. Tapirus terrestris (Linnaeus, 1758)

Localidad: MOCOA: Mocoa. ORITO: Río Caldero.

PUERTO LEGUízAMO: Parque Nacional Natural La Paya.

Distrito: Alto Putumayo; Kofán.

Eco-región: Bosques montanos de la Cordillera Oriental; Selva Húmeda del Napo.

Referencias: Mahecha et al., 1995; Polanco-Ochoa et al., 2000; Constantino et al., 2006; EANU, 2008: Observación personal.

Comentarios: esta especie es cazada por su carne principalmente para consumo familiar. Algunos ejemplares son cuidados en el Centro Experimental Amazónico, tras su decomiso a cazadores.

\section{ORDEN ARTIODACTYLA}

Familia Tayassuidae

148. Pecari tajacu (Linnaeus, 1758)

Localidad: ORITO: Río Caldero. PUERTO LEGUíZAMO: Parque Nacional Natural La Paya. Río Mecaya.

Distrito: Alto Putumayo; Kofán.

Eco-región: Selva Húmeda del Napo.
Referencias: FMNH 70565, FMNH 72412. Mahecha et al., 1995; Polanco-Ochoa et al., 2000; Rivas-Pava et al., 2007 (MHNUC 90E*); EANU, 2008: Observación personal.

149. Tayassu pecari (Link, 1795)

Localidad: ORITO: Río Caldero. PUERTO ASÍS: Bajo Río Guamués. Bocas del Río Peneya en la margen derecha del río Caquetá. PUERTO LEGUíZAMO: Parque Nacional Natural La Paya.

Distrito: Alto Putumayo; Kofán.

Eco-región: Selva Húmeda del Napo.

Referencias: ICN 4319, ICN 4331, ICN 4333, ICN 4338, ICN 4344, ICN 4350- 4351, ICN 4356, ICN 4358, ICN 5198, ICN 5199. Polanco-Ochoa et al., 2000; EANU, 2008: Observación personal.

\section{Familia Cervidae}

150. Mazama cf. americana (Erxleben, 1777)

Localidad: MOCOA: Mocoa. PUERTO ASÍS: Bajo Río Guamués. PUERTO LEGUíZAMO: Parque Nacional Natural La Paya.

Distrito: Alto Putumayo.

Eco-región: Bosques montanos de la Cordillera Oriental; Selva Húmeda del Napo.

Referencias: ICN 1557. Polanco-Ochoa et al., 2000; EANU, 2008: Observación personal.

151. Mazama nemorivaga $\mathrm{F}$. Cuvier, 1817

Localidad: ORITO: Río Caldero. PUERTO LEGUíZAMO: Parque Nacional Natural La Paya.

Distrito: Alto Putumayo; Kofán.

Eco-región: Selva Húmeda del Napo.

Referencias: FMNH 70558-70559. EANU, 2008: Observación personal.

Comentarios: Polanco-Ochoa et al., 2000, registraron esta especie como Mazama gouazoubira. Mazama nemorivaga fue considerada como un sinónimo de M. gouazoubira pero actualmente se tratan como especies parapátricas, con la última sin presencia en Colombia (Rossi \& Duarte, 2008). Es cazada por su carne principalmente para consumo familiar.

\section{ORDEN CETACEA}

Familia Iniidae

152. Inia geoffrensis (de Blainville, 1817)

Localidad: PUERTO LEGUíZAMO: Parque Nacional Natural La Paya.

Distrito: Alto Putumayo.

Eco-región: Selva Húmeda del Napo.

Referencia: Polanco-Ochoa et al., 2000. 


\section{Familia Delphinidae}

153. Sotalia fluviatilis (Gervais \& Deville, 1853)

Localidad: PUERTO LEGUíZAMO: Parque Nacional Natural La Paya.

Distrito: Alto Putumayo.

Eco-región: Selva Húmeda del Napo.

Referencia: Polanco-Ochoa et al., 2000.

\section{ORDEN SIRENIA}

\section{Familia Trichechidae}

154. Trichechus inunguis (Natterer, 1883)

Localidad: PUERTO LEGUÍZAMO: Parque Nacional Natural La Paya.

Distrito: Alto Putumayo.

Eco-región: Selva Húmeda del Napo.

Referencia:Polanco-Ochoa et al., 2000.

\section{Discusión}

Riqueza de especies, conservación y estado del conocimiento.

Aunque el número de especies de mamíferos registrados en el Putumayo abarca más del $30 \%$ del total registrado para Colombia, cabe resaltar que la mayor parte del Putumayo hace parte de la Amazonia, una de las áreas con mayor diversidad del mundo (Ceballos \& Ehrlich, 2006), por lo que se espera que la riqueza de mamíferos se incremente considerablemente con el desarrollo de nuevas investigaciones. Con respecto a los murciélagos que es uno de los grupos que mayor aporta a la riqueza de mamíferos en el país, el número de especies registrados (65) es menor en comparación con las 74 especies registradas en el departamento del Caquetá (Marín-Vásquez \& Aguilar-González, 2005), que es un territorio vecino al Putumayo y con ecosistemas afines.

Actualmente, los ecosistemas del Putumayo son el hábitat del mayor número de primates con distribución en Colombia (Defler, 2010) con 15 especies. Posiblemente, las condiciones típicas de ecotono (Lynch et al., 1997) entre ecosistemas de alta montaña (Andes) y selváticos (Amazonía), las gradiente altitudinales y la complejidad de la estructura vegetal (Defler, 2010), hacen del sur de Colombia hábitat óptimo para los primates y otras especies de mamíferos, por lo tanto esta región se convierte en un punto crucial para el desarrollo de investigaciones asociadas a este grupo. Los ecosistemas de la cuenca alta del Amazonas desde Nariño-Cauca hasta el sur del Putumayo-Caquetá, a diferencia del resto del país, presentan características particulares como los regímenes de humedad (muy húmedo en el norte, húmedo-sub-húmedos en el centro y húmedos en el sur), la disponibilidad hídrica y de materia orgánica en el suelo (Malagón, 2003), reflejándose en la continuidad de productos vegetales comestibles, recursos que posiblemente también explican la diversidad primates y otros mamíferos en el área.

El Putumayo y en general toda el área cubierta por las selvas amazónicas son el hábitat de 25 especies con alguna categoría de amenaza global o regional. En esas categorías los primates y carnívoros deben ser considerados objetos de conservación, por lo que se requiere desarrollar estudios para diseñar e implementar acciones que conlleven a la protección de dichos grupos. En la Amazonía la fuerte problemática ambiental a causa de la deforestación, ampliación de frontera agrícola, la contaminación por residuos químicos (Nepstad et al., 2011) y la presencia de cultivos de uso ilícito posiblemente ponen en riesgo un mayor número de especies que el reportado en este estudio.

En el departamento de Putumayo hay tres áreas protegidas presentes en el suroriente y norte del territorio (PNN, 2011), de los cuales solo existe información de la fauna de mamíferos de una (Polanco-Ochoa et al., 2000), por lo que es de gran urgencia generar información de la fauna presente en dichas áreas protegidas. Adicionalmente, se ha mencionado la necesidad de crear un Parque Nacional adicional en la parte suroccidental del Putumayo, dada la riqueza de primates que se encuentran en dicho sector (Defler, 2010).

La información publicada sobre los mamíferos de Putumayo no supera 20 trabajos, relacionados particularmente con los órdenes Chiroptera, Primates y Carnivora (Figura 3). Otros órdenes como Lagomorpha, Soricomorpha o Cingulata, han sido escasamente o no mencionados. Comparaciones con trabajos realizados para departamentos limítrofes como el Cauca (Ramírez-Chaves \& Pérez, 2011) y Nariño (Ramírez-Chaves \& Noguera-Urbano, 2011), muestran lo precaria de la investigación mastozoológica en el Putumayo y se resalta la necesidad de realizar mayor investigación en este sector del país.

\section{Distribución}

De los 336 registros para las 154 especies incluidas aquí (con una relación aproximada de dos registros por especie), 13 corresponden a un total de ocho localidades imprecisas. Generalmente datos imprecisos o no informativos constituyen fuentes de error al no permitir relacionar la presencia de una especie con el espacio geográfico y ambiental que habita (Ponder et al., 2001). Dentro de los meta-análisis y evaluación de la biodiversidad es necesaria la mayor cantidad de información georeferenciada posible (Peterson et al., 2010), por lo que esperamos que en futuros inventarios o muestreos, 
un punto clave a considerar sea tomar y reportar localidades precisas o georeferenciadas.

Muchos de los registros están concentrados en unos pocos municipios y unidades biogeográficas. Los registros se ubican en el noroccidente Putumayo distribuidos en ocho municipios de los 13 que integran el Putumayo, pero entre Puerto Leguízamo y Mocoa concentran el 63\% (202 registros) del total de registros con localidad precisas (320). La concentración de datos dentro de análisis de distribución implican sesgos (Ponder et al., 2001), que para el caso del departamento de Putumayo están asociados a una mayor cantidad de esfuerzos de muestreo en proximidades a carreteras y ríos, pero también problemas de orden público han hecho que no exista información proveniente de amplias áreas de dicho departamento. El ecosistema y Distrito con los mayores números de especies y registros fueron la Selva Húmeda del Napo y el Distrito Alto Putumayo respectivamente; las dos unidades también cubren el mayor terreno en el Putumayo e integran el mayor número de localidades (Figura 1).

La historia evolutiva de la biota y las condiciones ambientales presentes en el Putumayo, han sido reconocidas en la propuesta de los centros de endemismo Putumayo, Kofanes y Leguízamo (Hernández-Camacho et al., 1992b), sin embargo los autores no hacen mención alguna de especies que caractericen esos centros de endemismos. Según los resultados obtenidos, desde el noroccidente del Putumayo hay un gradiente que integra especies de Alta montaña, especies que se mueven entre tierras altas y bajas y especies de zonas bajas Amazónicas, por ejemplo los primates (Defler, 2010). Posiblemente, entre los distritos biogeográficos y las ecoregiones hay zonas de transición que necesitan ser evaluadas desde un enfoque biogeográfico; Lynch et al., (1997) han mencionado ecotonos o transiciones ecológicas en esta parte del país para explicar la alta diversidad y simpatría de especies. La información suministrada aquí puede servir como línea base para el desarrollo de propuestas de investigaciones enfocadas a incrementar el conocimiento sobre el importante grupo zoológico en el Putumayo. Sugerimos incrementar el número de muestreos, inventarios y estudios que permitan conocer mejor el estado actual de las poblaciones de mamíferos de esa parte de Colombia.

Dada, la heterogeneidad en el número de registros y especies de mamíferos que únicamente representan ocho municipios del departamento y unas pocas unidades biogeográficas, es necesario explorar áreas potenciales de estudio en municipios como Puerto Caicedo, Puerto Guzmán, San Francisco, Santiago y Colón o fomentar muestreos en diferentes gradientes altitudinales a lo largo de la cuenca del Rio Putumayo.

\section{Agradecimientos}

A los encargados de las colecciones del ICN (Yaneth MuñozSaba), IAvH (Juliana Castellanos, Fernando Forero y Diego Perico), MHNUC (Pilar Rivas), PSO-CZ (Jhon Jairo Calderón-Leyton), por permitir el acceso a la información de los ejemplares bajo su cuidado. HERC agradece a la Universidad de Queensland (becas UQCent y UQI) por el apoyo recibido durante las adecuaciones finales de este documento. EANU agradece a Profesores y estudiantes del Programa de Biología de la Universidad de Nariño, a funcionarios de la Corporación Autónoma de la Amazonia (CORPOAMAZONIA) y del Centro Experimental Amazónico (C.E.A.) por el apoyo recibido durante las exploraciones en campo; al posgrado en Ciencias de la Universidad Nacional Autónoma de México (UNAM) y al programa de becas 2011-2013 del Consejo Nacional de Ciencia y Tecnología de México (CONACyT), por el soporte dado para las adecuaciones finales de este documento. MRP agradece a Manuel Ruedi y a la ciudad de Ginebra Suiza por la financiación para la revisión de los especímenes de murciélagos colombianos depositados en el MHNG.

\section{Referencias}

Aellen, V. 1970. Catalogue raisonné des chiropterès de la Colombia. Revue Suisse de Zoologie 77 (1):1-37

Alberico, M., Cadena, A., Hernández-Camacho, J.I., Muñoz-Saba, Y. 2000. Mamíferos (Synapsida: Theria) de Colombia. Biota Colombiana 1 (1):43-75.

Anderson, R.P., Handley, Jr., C.O. 2001.A new species of three-toed sloth (Mammalia: Xenarthra) from Panamá, with a review of the genus Bradypus. Proceedings of the Biological Society of Washington 114 (1):1-33

Cadena, A., González-H., A. 2006. Murciélago blanco Diclidurusingens. En: J.V. Rodríguez-Mahecha, M. Alberico, F. Trujillo, J. Jorgenson (editores), Libro rojo de los mamíferos de Colombia. Conservación Internacional Colombia, Ministerio de Ambiente, Vivienda y Desarrollo Territorial, Bogotá D.C. 305-307 pp.

Ceballos, G., Ehrlich, P.R. 2006. Global mammal distributions, biodiversity hotspots, and conservation. Proceedings of the National Academy of Sciences 103 (51):19374-19379.

Constantino, E., Lizcano, D., Montenegro, O., Solano, C. 2006. Tapir de tierras bajas Tapirus terrestris. En: J.V. Rodríguez-Mahecha, M. Alberico, F. Trujillo, J. Jorgenson (editores), Libro rojo de los mamíferos de Colombia. Conservación Internacional Colombia, Ministerio de Ambiente, Vivienda y Desarrollo Territorial, Bogotá D.C. 106-112 pp.

Cropp, S.J., Larson, A., Cheverud, J. M. 1999. Historical biogeography of tamarins, genus Saguinus: The molecular phylogenetic evidence. American Journal of Physical Anthropology 108 (1):65-89.

Cuervo-Díaz, J., Hernández-Camacho, J.I., Cadena, A. 1986. Lista actualizada de mamíferos de Colombia: anotaciones sobre su distribución. Caldasia 15 (71-75):471-501.

Defler, T.R. 2004. Primates de Colombia. Tropical field guide series 5. Conservación Internacional. Bogotá- Colombia.

Defler, T.R. 2010. Historia natural de los primates de Colombia. Editorial Universidad Nacional de Colombia, Sede Bogotá, Bogotá. 
Defler, T.R., Rodríguez-M., J.V. 2006. Chichico diablo Callimico goeldii. En: J.V. Rodríguez-Mahecha, M. Alberico, F. Trujillo, J. Jorgenson (editores), Libro rojo de los mamíferos de Colombia. Conservación Internacional Colombia, Ministerio de Ambiente, Vivienda y Desarrollo Territorial, Bogotá D.C. 187-189 pp.

Defler, T.R., Palacios, E., Rodríguez, A. 2006. Mico volador Pithecia monachus. En: J.V. Rodríguez-Mahecha, M. Alberico, F. Trujillo, J. Jorgenson (editores), Libro rojo de los mamíferos de Colombia. Conservación Internacional Colombia, Ministerio de Ambiente, Vivienda y Desarrollo Territorial, Bogotá D.C. 232- 235 pp.

Díaz-N, J.F. 2012. New records of Marmosops noctivagus (Tschudi, 1845) (Didelphimorpia: Didelphidae) and first record of Marmosops bishopi (Pine, 1981) for Colombia. Check List 8 (4): 805-809.

Eger, J. 2008. Family Molossidae P. Gervais, 1856. En: A.L. Gardner (editor). Mammals of South America Volume 1.Marsupials, Xenarthrans, Shrews, and Bats. The University of Chicago Press, Chicago. 399$440 \mathrm{pp}$.

Gardner, A.L. (editor). 2008. Mammals of South America Volume 1. Marsupials, Xenarthrans, Shrews, and Bats.The University of Chicago Press, Chicago.

Groves, C. 2005. Order Primates. En: D. E. Wilson, D. M. Reeder (editors), Mammal Species of the World. Third Edition. Johns Hopkins University Press. Baltimore, USA. 111-184 pp.

Hernández-Camacho, J. 1955.Una nueva especie colombiana del género Diclidurus (Mammalia: Chiroptera): Diclidurus ingens. Caldasia 7 (31):87-98

Hernández-Camacho, J., Barriga-Bonilla, E. 1966. Hallazgo del genero Callimico (Mammalia, Primates) en Colombia. Caldasia 9 (44):365377.

Hernández-Camacho, J., Ortiz, R., Walsburger, T., Hurtado, A. 1992a. Unidades biogeográficas de Colombia. En: La Diversidad Biológica en Latinoamérica. Vol 1. G. Halffter. Acta Zoológica Mexicana Nueva Serie. Volumen especial. México. 105-152 pp.

Hernández-Camacho, J., Ortiz, R., Walsburger, T., Hurtado, A. 1992b. Centros de Endemismo en Colombia. En: La Diversidad Biológica en Latinoamérica. Vol 1. G. Halffter. Acta Zoológica Mexicana Nueva Serie. Volumen especial. México.175-190 pp.

Hershkovitz, P. 1963. A systematic and zoogeographic account of South American titi monkeys, genus Callicebus (Cebidae) of the Amazonas and Orinoco river basins. Mammalia 27 (1):1-80.

Hershkovitz, P. 1990. Titis, new world monkeys of the genus Callicebus (Cebidae, Platyrrhini): a preliminary taxonomic review. Fieldiana Zoology, New Series 55 (1410):1-109.

Hershkovitz, P. 1997. Composition of the family Didelphidae Gray, 1821 (Didelphoidea: Marsupialia), with a review of the morphology and behavior of the included Four-Eyed Pouched Opossums of the genus Philander Tiedemann, 1808. Fieldiana, Zoology, New series $\mathbf{8 6}$ (1485):1-102.

IGAC. 1996. Instituto Geográfico Agustín Codazzi. Diccionario Geográfico de Colombia. Tomo 3 Llacuanas-Quizgo. Tercera Edición. IGAC, Bogotá.

IUCN. 2012. IUCN Red List of Threatened Species. Version 2011.2. <http:// www.iucnredlist.org $>$. Descargado en Noviembrede 2012.

Jorgenson, J., Patiño, M., Silva, L. 2006a. Oso de Anteojos Tremarctos ornatus. En: J.V. Rodríguez-Mahecha, M. Alberico, F. Trujillo, J. Jorgenson (editores), Libro rojo de los mamíferos de Colombia. Conservación Internacional Colombia, Ministerio de Ambiente, Vivienda y Desarrollo Territorial, Bogotá D.C.243-248 pp.

Jorgenson, J., Rodríguez-Mahecha, J.V., Constantino, E., Barrera de Jorgenson, A. 2006b. Lobo colorado Lycalopex culpaeus. En: J.V.
Rodríguez-Mahecha, M. Alberico, F. Trujillo, J. Jorgenson (editores), Libro rojo de los mamíferos de Colombia. Conservación Internacional Colombia, Ministerio de Ambiente, Vivienda y Desarrollo Territorial, Bogotá D.C.237-241 pp.

Jorgenson, J., Rodríguez-M., J.V., Durán, C. 2006c. Puma Puma concolor. En: J.V. Rodríguez-Mahecha, M. Alberico, F. Trujillo, J. Jorgenson (editores), Libro rojo de los mamíferos de Colombia. Conservación Internacional Colombia, Ministerio de Ambiente, Vivienda y Desarrollo Territorial, Bogotá D.C.349-355 pp.

Lay, D.M. 1962. Seis mamíferos nuevos para la fauna de México. Anales del Instituto de Biología 33:373-377.

Lew, D., Pérez-Hernández, R., Ventura, J. 2006. Two new species of Philander (Didelphimorphia, Didelphidae) from northern South America. Journal of Mammalogy 87 (2):224-3.

Lim, B.K., Engstrom, M.D. 2001. Species diversity of bats (Mammalia: Chiroptera) in Iwokrama forest, Guyana, and the Guianan subregion: implications for conservation. Biodiversity and Conservation 10(4):613-657.

Lynch, J.D. Ruiz-C., P.M., Ardila-R., M.C. 1997. Biogeographic patterns of Colombian frogs and toads. Revista de la Academia Colombiana de Ciencias Exactas, Físicas y Naturales 21 (80):237-248.

Lizcano, D., Guarnizo, A., Suárez, J., Flores, F., Montenegro, O. 2006. Danta de páramo Tapirus pinchaque. En: J.V. Rodríguez-Mahecha, M. Alberico, F. Trujillo, J. Jorgenson (editores), Libro rojo de los mamíferos de Colombia. Conservación Internacional Colombia, Ministerio de Ambiente, Vivienda y Desarrollo Territorial, Bogotá D.C. 173-180 pp.

Malagón Castro, D. 2003. Ensayo sobre tipología de suelos colombianos -Énfasis en génesis y aspectos ambientales. Revista de la Academia Colombiana de Ciencias Exactas, Físicas y Naturales 27 (104):319-341.

MaNIS. 2012. MaNIS, the Mammal Networked Information System. Data downloaded from website http://manisnet.org/ (Marzo 2012).

Mantilla-Meluk, H., Jiménez-Ortega, A.M., Baker, R.J. 2009. Phyllostomid bats of Colombia: annotated chekclist, ditribution, and biogeography. Special Publications, Museum of Texas Tech University, Natural Science Research Laboratory, Texas Tech University 56:1-44.

Mantilla-Meluk, H., Ramírez-Chaves, H.E., Parlos J.A., Baker R.J. 2010.Geographic range extensions and taxonomic notes on bats of the genus Lonchophylla (Phyllostomidae) from Colombia.Mastozoología Neotropical 17 (2):295-303.

Marín-Vásquez, A., Aguilar-González, A.V. 2005. Murciélagos del departamento de Caquetá-Colombia. Biota Colombiana 6 (2):211-218.

Marinkelle, C.J., Cadena, A. 1971. Remarks on Sturnira tildae in Colombia.Journal of Mammalogy 52 (1):235-237.

Marinkelle, C.J., Cadena, A. 1972. Notes on bats new to the fauna of Colombia.Mammalia 36 (1):50-58.

Mueses-Cisneros, J.J. 2005. La fauna anfibia del Valle de Sibundoy, Putumayo-Colombia. Caldasia 27 (2):229-242.

Nepstad, D., McGrath D., Soares-Filho, B. 2011. Systemic Conservation, REDD, and the Future of the Amazon Basin. Conservation Biology 25 (6): $1113-1116$

Noguera-Urbano, E.A., Montenegro-Muñoz, S. 2011. Primer registro de la nutria tropical o lobito de río (Lontra longicaudis Olfers 1818) en el Piedemonte Andino-Amazónico y comentarios sobre la distribución en Putumayo Colombia. Novedades Colombiana 11 (1):19-25.

Olson, D.M., Dinerstein, E.,Wikramanayake, E.D., Burgess, N.D., Powell, G.V.N., Underwood, E.C., D’Amico, J.A., Strand, H.E., Morrison, J.C., Loucks, C.J., Allnutt, T.F., Lamoreux, J.F., Ricketts, T.H., Itoua, I., Wettengel, W.W., Kura, Y., Hedao, P., Kassem, K. 2001. Terrestrial ecoregions of the world: a new map of life on Earth. BioScience 51(11):933-938. 
Palacios, E., Defler, T.R., Rodríguez, J.V. 2006. Marimonda Ateles belzebuth. En: J.V. Rodríguez-Mahecha, M. Alberico, F. Trujillo, J. Jorgenson (editores), Libro rojo de los mamíferos de Colombia. Conservación Internacional Colombia, Ministerio de Ambiente, Vivienda y Desarrollo Territorial, Bogotá D.C. 215- 218 pp.

Peterson, A.T., Knapp S., Guralnick R., Soberón J., Holder M.T. 2010. The big questions for biodiversity informatics. Systematics and Biodiversity 8 (2):159-168.

Pinto, C.M., Carrera, J.P., Mantilla-Meluk, H., Baker, R.J.2007. Mammalia, Chiroptera, Phyllostomidae, Diaemus youngi: First confirmed record for Ecuador and observations of its presence in museum collections. Check List 3 (3):244-247.

PNN. 2011. Parques Nacionales Naturales de Colombia. Disponible en: http://www.parquesnacionales.gov.co (Julio de 2012).

Polanco-Ochoa, R., Jaimes, V., Piragua, W. [2000] “1999”. Los mamíferos del Parque Nacional Natural La Paya, Amazonía colombiana. Revista de la Academia Colombiana de Ciencias Exactas, Físicas y Naturales 23 (suplemento especial): 671-682.

Polanco Ochoa, R., López-Arévalo, H.F., Ángeles-Arce, M., Camargo, A.A. 2006. Oso hormiguero palmero Myrmecophaga tridactyla En: J.V. Rodríguez-Mahecha, M. Alberico, F. Trujillo, J. Jorgenson (editores), Libro rojo de los mamíferos de Colombia. Conservación Internacional Colombia, Ministerio de Ambiente, Vivienda y Desarrollo Territorial, Bogotá D.C. 182 pp.

Ponder, W.F., Carter, G.A., Flemons, P., Chapman, R.R. 2001. Evaluation of museum collection data for use in biodiversity assessment. Conservation Biology 15(3):648-657.

Ramírez-Chaves, H.E. 2008. Revisión taxonómica del género de murciélagos Eptesicus Rafinesque, 1820 (Mammalia: Chiroptera: Vespertilionidae), para Colombia. Tesis de Maestría, Universidad Nacional de Colombia.

Ramírez-Chaves, H.E., Pérez, W.A. 2011. Mamíferos (Mammalia: Theria) del departamento del Cauca, Colombia. Biota Colombiana 11 (1-2):141-171.

Ramírez-Chaves, H.E., Noguera-Urbano, E.A. 2011.Lista preliminar de los mamíferos (Mammalia: Theria) del departamento de Nariño, Colombia. Biota Colombiana 11 (1-2):117-140.

Ramírez-Chaves, H.E., Ortega-Rincón, M., Pérez, W. A., Marín, D. 2011. Historia de las especies de mamíferos exóticos en Colombia. Boletín Cientifico, Centro de Museos, Universidad de Caldas 15 (2):139-156

Riascos, J.M. 2001. Aves, Mamíferos, Reptiles y Anfibios de la Colección Zoológica de Referencia "IMCN" del Museo de Ciencias Naturales Federico Carlos Lehmann V. Cespedesia 24 (75-78):95-152.

Rivas-Pava, M., Ramírez-Chaves, H.E., Álvarez, Z., Niño-Valencia, B. 2007. Catálogo de los mamíferos presentes en las colecciones de referencia y exhibición del Museo de Historia Natural de la Universidad del Cauca. Taller Editorial Universidad del Cauca, Popayán.

Rodríguez-Mahecha, J.V., Hernández-Camacho, J.I., Defler, T.R., Alberico, M., Mast, R.B., Mittermeier, R.A., Cadena, A. 1995. Mamíferos colombianos: sus nombres comunes e indígenas. Occasional papers in Conservation Biology No. 3. Conservation International, Bogotá, Colombia.

Rodríguez-Mahecha, J., Alberico, M., Trujillo, F., Jorgenson, J. (editores) 2006a. Libro rojo de los mamíferos de Colombia. Conservación Internacional Colombia, Ministerio de Ambiente, Vivienda y Desarrollo Territorial, Bogotá D.C.

Rodríguez, A., Palacios, E., Defler, T.R., Rodríguez-Mahecha, J.V. 2006. Mico tocón Callicebus cupreus. En: J.V. Rodríguez-Mahecha, M. Alberico, F. Trujillo, J. Jorgenson (editores), Libro rojo de los mamíferos de Colombia. Conservación Internacional Colombia, Mi- nisterio de Ambiente, Vivienda y Desarrollo Territorial, Bogotá D.C. 226-230 pp.

Rodríguez-Posada, M.E., Sánchez-Palomino, P. 2009. Taxonomía del género Phyllostomus (Chiroptera: Phyllostomidae) en Colombia. Mastozoología Neotropical 16 (1):153-168.

Rossi, R.V., Duarte, J.M.B. 2008. Mazama nemorivaga. En: IUCN 2011. IUCN Red List of Threatened Species. Version 2011.2. www.iucnredlist.org. Accedido el 07 de Mayo de 2012.

Rossi R.V., Voss, R.S., Lunde, D.P. 2010. A revision of the Didelphid marsupial genus Marmosa. Part 1.The species in Tate's 'mexicana' and 'mitis'sections and other closely related forms. Bulletin of the American Museum of Natural History 334:1-83

Santa Gertrudis, F.J.D. 1956 [1775]. Maravillas de la naturaleza. Empresa Nacional de Publicaciones, Bogotá.

Steppan, S.J. 1995. Revision of the tribe Phyllotini (Rodentia: Sigmodontinae), with a phylogenetic hypothesis for the Sigmodontinae. Fieldiana Zoology, New Series 80 (1464):1-112.

Stevenson, P.R., Pérez-Torres, J., Muñoz-Saba, Y. 2006. Estado del conocimiento sobre los mamíferos terrestres y voladores de Colombia. En: M.E.Chaves, M. Santamaría (editores), Informe nacional sobre el avance en el conocimiento y la información de la biodiversidad 1998-2004. Tomo II. Instituto de Investigación de Recursos Biológicos Alexander von Humboldt. Bogotá D.C. 151-170 pp.

Tamsitt, J.R., Cadena, A., Villarraga, E. 1986. Records of bats (Sturnira magna and Sturnira aratathomasi) from Colombia. Journal of Mammalogy 67(4):754-757.

Trujillo, F., Díazgranados, M.C., Caicedo-Herrera, D. 2006. f. Mamíferos de agua dulce. En: M.E.Chaves, M. Santamaría (editores), Informe nacional sobre el avance en el conocimiento y la información de la biodiversidad 1998-2004. Tomo II. Instituto de Investigación de Recursos Biológicos Alexander von Humboldt. Bogotá D.C. 230-240 pp.

Veiga, L.M., Palacios, E. 2008. Callicebus medemi. En: IUCN 2011. IUCN Red List of Threatened Species.Version 2011.2. <www.iucnredlist. org $>$. Downloaded on 21 March 2012.

Velazco, P.M. 2005. Morphological Phylogeny of the bat Genus Platyrrhi$n u$ S Saussure, 1860 (Chiroptera: Phyllostomidae) with the description of four new species. Fieldiana Zoology, New Series 105(1535):1-53.

Velazco, P.M., Gardner, A.L. 2009. A new species of Platyrrhinus (Chiroptera: Phyllostomidae) from western Colombia and Ecuador, with emended diagnoses of P. aquilus, P. dorsalis, and P. umbratus. Proceedings of the Biological Society of Washington 122(3):249-281.

Velazco, P.M., Simmons, N.B. 2011. Systematics and taxonomy of Great Striped-Faced bats of the genus Vampyrodes Thomas, 1900 (Chiroptera: Phyllostomidae). American Museum Novitates 3710:1-35

Velazco, P.M., Gardner, A.L., Patterson, B.D. 2010. Systematics of the Platyrrhinus helleri species complex (Chiroptera: Phyllostomidae), with descriptions of two new species. Zoological Journal of the Linnean Society 159(3): 785-812.

Weksler, M., Percequillo, A.R., Voss, R.S. 2006. Ten new genera of Oryzomyine rodents (Cricetidae: Sigmodontinae). American Museum Novitates 3537:1-29.

Williams, S.L., Willig, M.R., Reid, F.A. 1995. Review of the Tonatia bidens complex (Mammalia: Chiroptera), with descriptions of two new subspecies. Journal of Mammalogy 76(2):612-626.

Wilson D.E., Reeder, D.M. (editors.). 2005. Mammal Species of the World. Third Edition. Johns Hopkins University Press. Baltimore.

Recibido: 3 de mayo de 2013

Aceptado para su publicación: 4 de junio de 2013 